\title{
Alexander von Humboldt oder die Suche nach dem Ganzen
}

Wir stehen jetzt vor einem gewaltigen Oeuvre, dem gesamten Schaffen von Alexander von Humboldt, das uns schon auf einer rein quantitativen Ebene wirklich erschrecken kann. Aber keine Angst! Stellen Sie sich einfach einen Menschen vor, der durch sukzessive Schlafreduktion ein Leben lang mit drei bis vier Stunden Schlaf auskommt und fast neunzig Jahre alt wird. Und dieser Mensch schreibt, er schreibt in allen Situationen und Lebenslagen. So entstehen Abertausende von Seiten publizierter Werke - allein das Opus Americanum umfasst vierunddreißig Bände -, Abertausende von Seiten eines bis heute noch lange nicht veröffentlichten handschriftlichen Nachlasses, allein viereinhalbtausend Seiten seiner Amerikanischen Reisetagebücher - an denen wir an der Akademie mit einem Akademienvorhaben noch für anderthalb Jahrzehnte beschäftigt sein werden - und mehr als vierzig- bis fünfzigtausend Briefe, von denen rund die Hälfte mittlerweile veröffentlicht ist. Wahrhaftig ein gewaltiges Oeuvre!

Aber wir brauchen uns dem Jüngeren der beiden Humboldt-Brüder nicht mit Ehrfurcht oder Schaudern anzunähern. Denn alles bei Alexander von Humboldt ist leicht und einfach. So lässt sich sein Leben, das an einem 14. September 1769 im Zeichen eines Kometen begann und am 6. Mai 1859 fast neunzig Jahre später ebenfalls in Berlin endete, in drei Epochen von jeweils knapp dreißig Jahren einteilen. Sie verstehen schon: Die Neunerjahre sind HumboldtJahre, da gibt es immer einen Jahrestag zu begehen und zu feiern.

Diese drei Epochen oder Phasen seines Lebens werden jeweils durch die großen transkontinentalen Reisen, die er unternahm, rhythmisiert und miteinander verbunden. Die erste, die berühmte amerikanische Reise, dauerte von 1799 bis 1804 und führte ihn in die heutigen Länder Venezuela, Cuba, Kolumbien, Ecuador, Peru und Mexico sowie abschließend noch einmal nach Cuba und dann ganz kurz in die Vereinigten Staaten von Amerika, bevor es wieder nach Frankreich zurückging. Die zweite große Reise brachte Humboldt im Jahre 1829 von Berlin quer durch das russische Zarenreich bis an die chinesische Grenze, so dass der amerikanischen eine russisch-sibirische Forschungsreise gegenübersteht, die sich komplementär zur erstgenannten verhält. Alles entstand mit gütiger Hilfe des Zufalls, aber deshalb keineswegs planlos. Denn verlief die amerikanische Reise innerhalb der Tropen Amerikas von Norden nach Süden und wieder nach Norden, so führte Humboldt seine Reise ins Innere Asien von Westen nach Osten und wieder nach Westen - und zwar außerhalb der Tropen. 


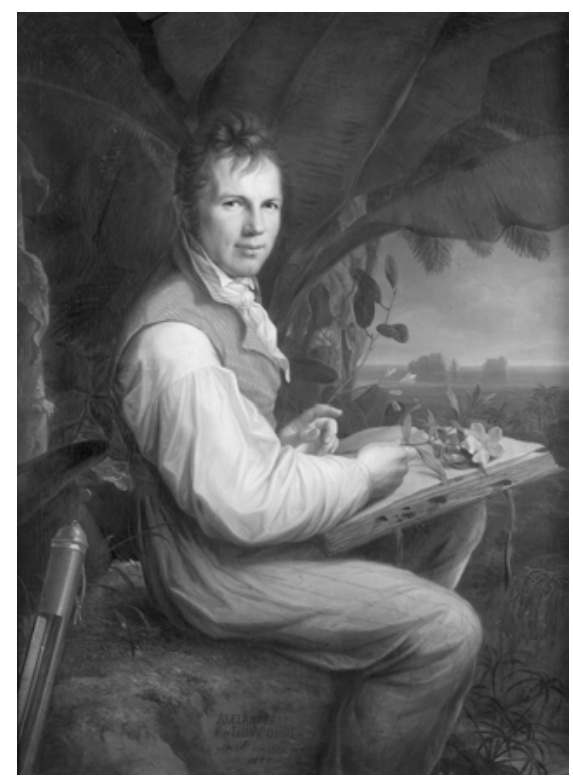

Abb. 71: Alexander von Humboldt (Berlin, 1769 - ebenda, 1859).

Allein schon aufgrund seiner auch sonst sehr regen Reisetätigkeit war Humboldt eine Ausnahmefigur. Es gibt nur wenige Zeitgenossen, die so akribisch genau einen großen und zudem repräsentativen Teil der Erdoberfläche in ihrem Leben im 19. Jahrhundert besuchten und untersuchten. Die übrigen 'kleineren' Reisen nach Polen oder Österreich, in die Schweiz oder nach England, nach Italien oder Frankreich lassen wir hier einfach weg.

Zur ersten Phase seines Lebens ist zu sagen, dass er auf Betreiben der Mutter zusammen mit seinem älteren Bruder Wilhelm von den besten Hauslehrern unterrichtet wurde, die in Berlin aufzutreiben waren. Er arbeitete sich in die unterschiedlichsten Gegenstände ein, studierte in Frankfurt an der Oder - Berlin hatte noch keine Universität - und in Göttingen, lernte in Hamburg und an der Bergakademie in Freiberg und realisierte schließlich eine Blitzkarriere im preußischen Bergdienst, die er erst dann für einige überraschend abbrach, als seine Mutter verstarb.

Dann begann ein neues Leben. Denn das - wie wir heute sagen könnten Millionenerbe legte er nicht wie sein Bruder überwiegend an, sondern verwendete es für seine Reisen und die nachfolgenden Publikationen, steckte sein Geld folglich in die Wissenschaft, mit der er eine Full-time-Beziehung pflegte. Er veröffentlichte Bücher und Aufsätze in den unterschiedlichsten Gebieten und Disziplinen in dieser ersten Phase, von der Geologie über die kryptogamen 


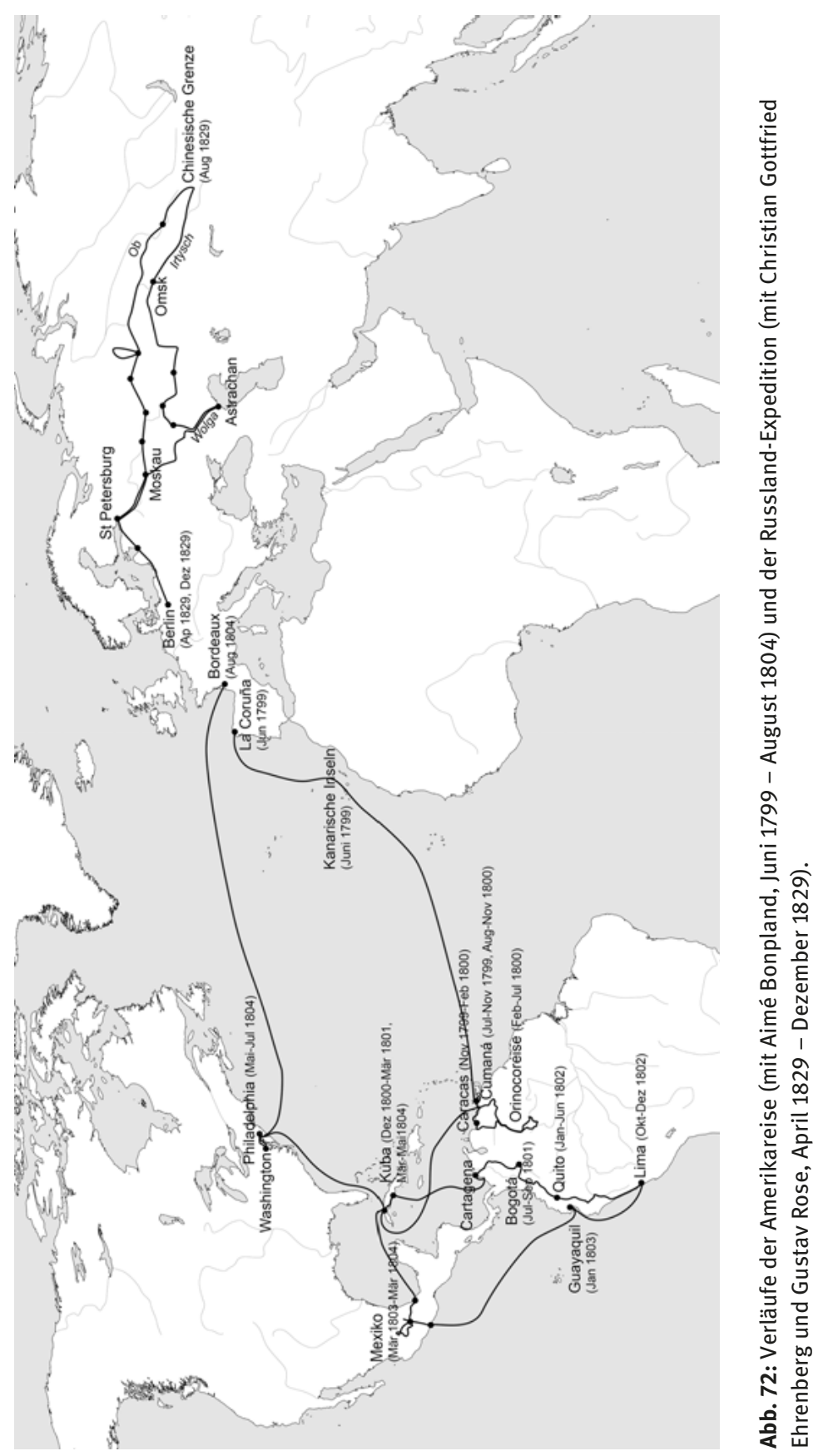


Gewächse in Freiberger Stollen bis hin zur gereizten Nervenfaser, führte Selbstversuche durch und gönnte sich kaum einmal Ruhe. Humboldt war ein Hansdampf in allen Gassen.

Am Abschluss dieser Phase aber steht dann die Reise in die amerikanischen Tropen. Die Amerikanischen Reisetagebücher werden zum Geburtszeugnis seiner Humboldt'schen Wissenschaft, die auf der Reise geradezu notwendig entsteht: Wir schauen Humboldt in diesen Reisetagebüchern gleichsam über die Schulter und können zusehen, wie aus den Einzeldisziplinen eine hochvernetzte Gesamtheit erwächst, die transdisziplinär verbunden und vernetzt ist und Natur und Kultur, Naturwissenschaft und Kulturwissenschaft, auf höchst originelle Weise zusammendenkt. Es ist ein Wissenschaftsentwurf, der auch für uns noch Zukunft hat.

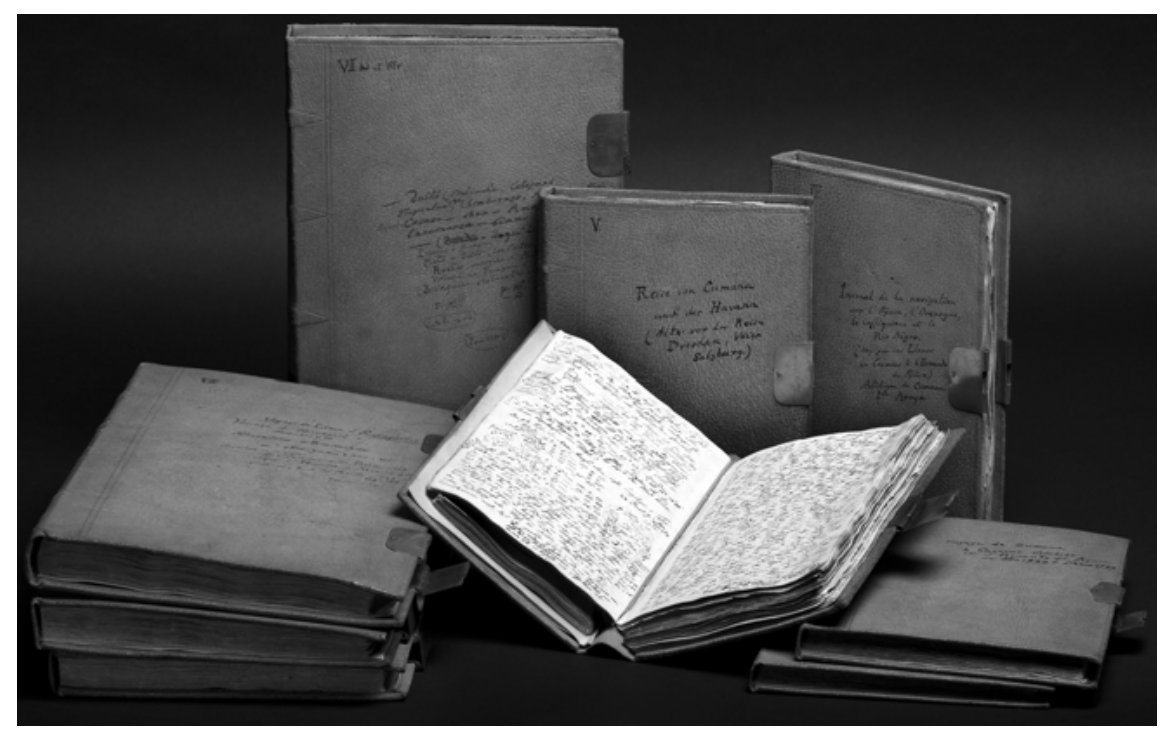

Abb. 73: Die 4.000 eng beschriebenen Seiten der Amerikanischen Reisetagebücher Alexander von Humboldts.

In der zweiten Phase seines Lebens, die mit seiner Rückkehr nach Europa beginnt, lebt Humboldt vorwiegend in Paris, von wo aus er die wissenschaftliche Aufarbeitung seiner Reise in Angriff nimmt. Paris ist zum damaligen Zeitpunkt das wichtigste wissenschaftliche Zentrum weltweit - und Humboldt, der schon vor seiner Reise beste Kontakte zu französischen Wissenschaftlern entwickelt 
hatte, versteht dieses Zentrum für sich und seine Wissenschaft zu nutzen. Er wird $\mathrm{zu}$ einem französischen Schriftsteller und Wissenschaftler, veröffentlicht den größeren Teil seiner Werke auf Französisch, besucht aber auch die Pariser Salons, wo er ein nicht nur geschätzter, sondern gefeierter - und aufgrund seiner spitzen Zunge auch gefürchteter - illustrer Besucher ist. Nein, anders als Casanova eroberte er hier nicht eine Unzahl an Frauenherzen: Humboldt war in erster Linie mit seiner Wissenschaft verheiratet.

Als er 1827 auf nachdrückliches königliches Bitten hin nach Preußen zurückkehren muss, um seine schon formell langjährige Tätigkeit als Kammerherr am Hofe Friedrich Wilhelms III. persönlich auszuüben, da verwandelt er Berlin in ein Wissenschafts-Zentrum, indem er mit Hilfe des Hofes wie der Akademie der Wissenschaften eine bärenstarke Wissenschaftspolitik betreibt. Alexander von Humboldt mausert sich zum Wissenschaftsorganisator. Er hält nicht nur sechzig Vorlesungen an der inzwischen gegründeten Berliner Universität, sondern auch sechzehn sogenannte 'Kosmos-Vorträge' im größten Saal Berlins, in der Berliner Singakademie, dem heutigen Maxim-GorkiTheater. Damit erreicht er breite Schichten der Bevölkerung und vor allem auch die Frauen, die noch lange aus der Universität in Preußen ausgesperrt bleiben sollten. Humboldt vertritt eine demokratische Auffassung von Wissenschaft. Oder anders formuliert: Mit dem Konzept der Open Science wäre er heutzutage mehr als einverstanden.

Die dritte Phase beginnt dann mit der russisch-sibirischen Forschungsreise, die auf Einladung des Zaren erfolgt und in gewisser Weise staatlich organisiert wird. Dadurch sind die gewaltigen Distanzen innerhalb des Russischen Reiches binnen kürzester Zeit überwindbar: Alles ist bestens organisiert. Doch es gibt auch eine Kehrseite: Humboldt hat sich freilich dafür verpflichten müssen, die politischen Dinge nicht anzusprechen - eine Verpflichtung, an die er sich in seinen Veröffentlichungen weitgehend hält. Dass er sich außerhalb seiner Veröffentlichungen etwa für verfolgte und unterdrückte Polen einsetzt, ist Ehrensache. Völlig ließ sich ein Humboldt den Mund nicht verbieten.

Als er von der Reise, auf der er die Entdeckung von Diamanten vorhergesagt hat - eine Vorhersage, die dann tatsächlich eintrifft -, wieder zurück in Berlin ist, beginnt der Abschluss des amerikanischen Reisewerkes, der Abschluss des asiatischen Reisewerkes und schließlich die Arbeit an seiner Summa, dem Kosmos, an dem er bis zu seinem Lebensende sitzen und unaufhörlich schreiben sollte. Als er schließlich mit fast neunzig Jahren in Berlin verstirbt, ist er ganz ohne Zweifel zu jenem Zeitpunkt der berühmteste Wissenschaftler seiner Epoche. Sein Lebenswerk, an dessen Edition wir heute wieder sitzen, ist von monumentaler Größe. 
Aber wir dürfen ihn uns nicht so sehr als ein Monument, ja als einen Monolithen vorstellen. Denn Humboldt ist ein Mensch; und als Mensch von einer ungeheuren Dynamik, die alle Teile seines Lebens erfasst und vieles mit sich reißt. Er ist beständig mit allen möglichen Aktivitäten beschäftigt und bewegt sich pausenlos zwischen verschiedenen Aufgaben hin und her. Es ist eine Wissenschaft aus der Bewegung, die eine geradezu kubistische Sichtweise der Dinge entwickelt, indem sie die Phänomene gleichzeitig aus verschiedenen Perspektiven betrachtet. Nähern wir uns also der Vielgestaltigkeit und Vielverbundenheit eines immensen Werkes an, das aus der Bewegung entstand und unter einem einfachen, von ihm auf der amerikanischen Reise niedergeschriebenen Leitaxiom als Humboldt'sche Wissenschaft stand: 'Alles ist Wechselwirkung'. Der vielleicht konziseste und auf einen einzigen Blick erfassbare Gesamteindruck dieses Wissenschaftskonzepts ist eines der berühmtesten Wissenschaftsbilder des 19. Jahrhunderts, Humboldts Tableau physique, sein 'Naturgemälde der Tropenländer', das Kunst und Wissenschaft, Ästhetik und Ethik miteinander eindrucksvoll vernetzt (vgl. Abb. 25: „Tableau physique des Andes et pays voisins“, Alexander von Humboldt, 1805).

An einem 3. Januar des Jahres 1806 schickte Alexander von Humboldt seinem Schweizer Freund, dem Naturforscher Marc-Auguste Pictet, einen kurzen, bislang in der Forschung nur selten berücksichtigten (geschweige denn eingehender untersuchten) Text, dem er - scherzhaft und hintergründig wie stets den Titel Mes confessions gab. Diese in französischer Sprache verfassten 'Bekenntnisse' sollten dem einflussreichen Pictet dabei behilflich sein, für die von Humboldt ins Auge gefasste und mit mancherlei Hoffnungen nicht zuletzt finanzieller Art ersehnte englischsprachige Ausgabe seines Reisewerks kräftig die Werbetrommel zu rühren. Denn Humboldt wusste, dass die Werbung für die eigene Sache in der Wissenschaft immer grundlegend wichtig ist: 'Läuten gehört zum Handwerk', wie er sich auszudrücken pflegte.

Gegen Ende dieses eindrücklichen autobiographischen Textes 'bekannte' der damals sechsunddreißigjährige Forscher, der bereits vor seiner Reise nach Amerika als Wissenschaftler international anerkannt und nach seiner Rückkehr zu einer europäischen Berühmtheit geworden war:

Inquiet, agité et ne jouissant jamais de ce que j'ai achevé, disait-il de lui-même, je ne suis heureux qu'en entreprenant du nouveau et en faisant trois choses à la fois. C'est dans cet esprit d'inquiétude morale, suite d'une vie nomade, que l'on doit chercher la cause principale de la grande imperfection de mes ouvrages. J'aurai été plus utile par les choses et les faits que j'ai rapportés, par les idées que j'ai fait naître dans d'autres, que par les ouvrages que j'ai publié moi-même. Cependant je n'ai pas manqué ni de bonne et de grande volonté, ni d'assiduité au travail. Dans les climats les plus ardents du globe, j’ai écrit ou 
dessiné souvent 15 à 16 heures de suite. Ma santé n'en a pas soufferte, et je me prépare au voyage d'Asie après avoir publié les résultats du voyage d'Amérique. ${ }^{1}$

Dies ist zweifellos eine ganz wunderbare Selbstdarstellung, welche die Unruhe, die kreative Unruhe, Alexander von Humboldts sehr deutlich werden lässt, verbunden mit seiner Tatkraft und einer gewissen Bescheidenheit zu einem Zeitpunkt, als freilich noch nichts von seinem Opus Americanum veröffentlicht war: Gerade erst waren die ersten Teile desselben fertiggestellt. Mag uns auch manch sprachliche Unkorrektheit oder Ungeschicklichkeit dieses französischsprachigen Textes daran erinnern, dass Alexander ihn nicht für die Publikation vorgesehen, sondern als einen Gebrauchstext konzipiert hatte, dem man ihm „eines Tages wieder zurücksenden“ solle, so wirft er doch ein bezeichnendes Licht auf den großen Naturforscher, Gelehrten und Schriftsteller.

Denn jene „Gemütsverfassung moralischer Unruhe“2 verweist uns - in der Verdoppelung des Lexems inquiet - auf eine Ruhelosigkeit, die stets in die $\mathrm{Zu}$ kunft gerichtet und damit prospektiv ist. Sie erscheint ihm als Folge eines Nomadenlebens, welches einer nur vorübergehenden Sesshaftigkeit Platz gemacht hat, so dass für Alexander von Humboldt die Zeit nach der Reise längst wieder zur Zeit vor der Reise geworden ist. Alexander von Humboldt war stets ein Mensch auf dem Sprung.

Dass der so ungeduldig erwartete Aufbruch nach Asien erst drei Jahrzehnte nach dem Beginn seiner Reise in die Äquinoktial-Gegenden des Neuen Kontinents und nicht in der ursprünglich von ihm geplanten Form erfolgen würde, konnte der mit der europäischen République des Lettres wohlvertraute Jüngere der beiden Humboldt-Brüder damals noch nicht ahnen. Er unterschätzte ganz offensichtlich den Aufwand der Fertigstellung seines amerikanistischen Werkes, das er in der Tat erst dreißig Jahre später mit dem Examen critique abschließen sollte.

Humboldts Bekenntnisse erfassen auf den ersten Blick nur jene erste Phase seines Lebens einschließlich seiner Reise in die Tropenwelt Amerikas, die sein Bild in der europäischen wie außereuropäischen Öffentlichkeit in der Folge so sehr bestimmen sollte. Doch ist jener esprit, den wir nur unbefriedigend mit dem bis ins 20. Jahrhundert wahrlich strapazierten deutschen Gegenbegriff

\footnotetext{
1 Humboldt, Alexander von: Mes confessions. In: Le Globe (Genève) 7 (janvier - février 1868), S. 180-190. Eine deutschsprachige Übersetzung findet sich erstmals in Humboldt, Alexander von: Meine Bekenntnisse (Autobiographische Skizze 1769-1805). In (ders.): Aus meinem Leben. Autobiographische Bekenntnisse. Zusammengestellt und erläutert von Kurt-R. Biermann. München: Beck 1987, S. 49-62.

2 Ebda., S. 60.
} 
'Geist' übersetzen könnten, ein Charakteristikum nicht nur der ersten Phase, sondern Ausgangspunkt seiner unermüdlichen Aktivitäten überhaupt und Grundbedingung eines Schreibens in der Moderne, für das die Humboldt'sche écriture und dies ist bislang noch kaum gesehen worden - geradezu modellhaft und exemplarisch steht.

Noch einmal kurz zum Textstatus: Vordergründig dienen Mes confessions als Grundlage für eine Werbekampagne. Doch wie sollte man für die Veranstaltung einer Ausgabe mit der Aussage werben, dass diese Werke eine „große Unvollkommenheit“ aufweisen? Wie sollte man Verleger für einen Autor gewinnen, der von sich selbst behauptet, weniger durch die von ihm bislang publizierten Werke als durch jene Ideen gewirkt $\mathrm{zu}$ haben, die er bei anderen angeregt habe?

Nicht von ungefähr verweist Alexander von Humboldt auf ein Schreiben (und ein Zeichnen), das er selbst unter widrigsten Bedingungen und in ungeheurer Anstrengung unermüdlich und fleißig während der Reise selbst vorangetrieben habe, jenes 'Schreiben im Angesicht der Dinge', das für seine Wissenschaftskonzeption so entscheidend ist. Mithin springt das Nomadenleben auf das Schreiben über, aus der vie nomade wird eine écriture nomade, die nicht nur den Ort des Schreibens, sondern auch dessen Gegenstände ständig gegen andere vertauscht. Bewegung ist der Schlüssel zu Alexander von Humboldts gesamtem Leben und Werk.

Wie so häufig saß Humboldt bei der Verfassung seines Textes ein wenig der Schalk im Nacken. Die Anspielung auf Jean-Jacques Rousseaus Les Confessions, mit denen der citoyen de Genève die moderne Autobiographie ${ }^{3}-$ und vielleicht mehr noch die Autobiographie in der Moderne - eröffnete, ist im Schreiben an den gelehrten Genfer Freund Pictet nicht nur ein ironisches Augenzwinkern, sondern zugleich auch Selbstvergewisserung und Selbstreflexion, die aus der Erfahrung des Erfolgs keine Statik, sondern nur noch weiteren Antrieb, zusätzlich beschleunigte Bewegung gewinnt. Der unmittelbar zuvor erfolgte Hinweis auf seine Aufnahme ins Pariser Institut National, die Berliner Akademie der Wissenschaften oder die American Philosophical Society in Philadelphia benennt die äußeren Zeichen, nicht aber die Bedingungen eines Erfolges, in dem sich das Glücklichsein nur in der Form des Künftigen, des Projektierens und Projizierens, einstellen wollte. Humboldt schrieb und lebte stets in einem prospektiven Sinne, in Gedanken und im Denken an die Zukunft.

3 Vgl. die Überlegungen zur Gattungsentwicklung in Jurt, Joseph: Mauriac face au créateur de l'autobiographie moderne: J.-J. Rousseau. In: Durand, François (Hg.): Mauriac et l'autobiographie. Paris: Grasset 1990, S. 135-148. 
Sind Mes Confessions vor allem eine Selbststilisierung? Sicherlich nicht. Mit einer spanischen Redewendung ließe sich sagen, dass Humboldt gleichsam die Etappen seines Schaffens 'abbrannte', stets vom Gegenwärtigen zum Künftigen eilte und sich dabei der Schrift, die doch auch das Überzeitliche, das Andauernde repräsentiert, nur in dieser eilenden Bewegung widmen zu können glaubte. Humboldt war, wie gesagt, stets auf dem Sprung: bereit für das Künftige, das Kommende. Dies gilt letztlich auch für seine gesamte Wissenschaftskonzeption.

Gewiss: Er konzentrierte sich jahrzehntelang auf die Ausarbeitung nicht nur der Ergebnisse seiner Reise und ließ dabei das Wissen der unterschiedlichsten Disziplinen in seine Schriften einfließen. Doch sollte sein Oeuvre stets modern gesprochen - ein work in progress bleiben, eine projektierte Totalität, die sich in gigantischen Fragmenten, in ungeheuren Proliferationen der Schrift entlud. Humboldt schrieb nicht etwas zusammen, er dachte alles zusammen und in großen Zusammenhängen. Ihm war die Vorläufigkeit jedes einmal erreichten Wissensstandes dabei sehr wohl bewusst. Dieses geschärfte Bewusstsein des Transitorischen avancierte zur eigentlichen Bedingung ständiger Bearbeitungen seiner eigenen Schriften und damit weiterer Gebiete, die sich sein nomadisierendes Schreiben in einem expansiven Prozess einverleibte. Jede Überarbeitung ist bei Humboldt eine Erweiterung.

Angesichts einer derartigen Konzeption des Schreibens überrascht es nicht, dass Alexander von Humboldts Korrespondenz zu den umfangreichsten - von seinen wohl an die fünfzigtausend Briefen sind etwa an die zwanzigtausend erhalten geblieben - und vor allem bedeutungsvollsten Epistolarien seiner Zeit gehört, sind Briefe doch gerade jenes Medium, in dem sich ständige Wechsel des Ortes, der Zeit und der Gegenstände vielleicht am unmittelbarsten auszudrücken vermögen. Die weitgespannte Korrespondenz erlaubte ihm, in relativ schneller Folge Erkenntnisse und Ergebnisse auszutauschen, panoramatische Sichtweisen gleichsam fragmentartig $\mathrm{zu}$ entwickeln und mit Ideen $\mathrm{zu}$ experimentieren, die ihm in späteren Publikationen noch als nützlich erscheinen würden. Auch wenn die Briefe Humboldts in der Regel lang waren, so erlaubten sie ihm doch, in jenen relativ kurzen Einheiten zu schreiben, aus denen sich sein Werk zusammensetzt. Denn Humboldt schrieb sehr gerne in Text-Inseln.

Präsentierte sich Rousseau gleich zu Beginn seiner Bekenntnisse am Tage des Jüngsten Gerichts vor seinem Richter „mit diesem Buch in meiner Hand“, ${ }^{4}$

4 Rousseau, Jean-Jacques: Les Confessions. In (ders.): Oeuvres complètes. Bd. I. Edition publiée sous la direction de Bernard Gagnebin et Marcel Raymond avec, pour ce volume, la collaboration de Robert Osmont. Paris: Gallimard 1959, S. 5: „Möge die Trompete des Jüngsten Gerichts erschallen, wann immer sie wolle; ich werde mit diesem Buch in meiner Hand vor dem höchsten Richter erscheinen.“ 
so verzichtete auch Humboldt in seinen Bekenntnissen nicht auf eine Rechtfertigung seines Tuns; doch ließe sich Rousseaus auf das eigene Buch gerichtete Geste - „Seht, was ich getan habe, was ich gedacht habe, was ich war“5 - nicht ohne weiteres ${ }^{6}$ auf den jungen preußischen Schriftsteller und Gelehrten übertragen. Alexander warf, anders als Jean-Jacques, volonté und assiduité au travail in die Waagschale, (nicht nur preußische) Tugenden, die jenseits der Genieästhetik stehen. Sie sind Garanten eines nomadischen Schreibens, das in der zentrifugalen Bewegung moralischer Unruhe sein paradoxes Zentrum besitzt. Humboldts Kontinuität war die Unruhe - oder wie bei einer Uhr die Unruh.

In Humboldts Bekenntnissen schlägt ein autobiographisches Schreiben durch, das sich- seiner selbst in unsteter Bewegung sich versichernd - ganz im Sinne des Rousseau'schen Modells in ein erzählendes und ein erzähltes Ich aufspaltet. Wir haben auf dieser Ebene die Parallelen zwischen der modernen Autobiographie und dem (modernen) Reisebericht in unserer Vorlesung bereits beleuchtet. Die Spannung entsteht nicht nur aus der Wechselbeziehung zwischen beiden, sondern auch aus der Tatsache, dass das erzählende Ich in der Form des zurückblickenden, schreibenden Ich (sehr im Gegensatz zu einem seiner narrativen Taufpaten, dem pícaro) nicht zur Ruhe gekommen ist. Es gewinnt seine Beobachterposition paradoxerweise aus der übergroßen Geschwindigkeit, die - wie wir nicht erst seit der Erfahrung der Jets wissen ${ }^{7}$ - in ein prekäres Gefühl der Ruhe umzukippen neigt. So wird der Beobachtungsstand zu einem Beobachtungspunkt im Vektorenfeld einer Bewegung, deren Ziel sich immer von neuem erst ergibt, ganz wie der Horizont vor dem voranschreitenden Wanderer immer weiter zurückweicht. Für Humboldt war das Reisen geradezu ein Seins-Zustand.

Diese autobiographische Erzählsituation beschränkt sich bei Humboldt aber keineswegs auf die autobiographischen Skizzen oder seine ausgedehnte Korrespondenz, sondern umfasst über seine reiseliterarischen Texte hinaus - wenn auch in unterschiedlicher Stärke - sein gesamtes Schaffen, sein gesamtes Werk. Die für das friktionale Genre des literarischen Reiseberichts der Moderne gattungskonstitutive Aufspaltung in erzähltes und erzählendes Ich ist bei Humboldt aber nicht deckungsgleich mit der Trennung zwischen reisendem und schreibendem Ich. Das reisende Ich schreibt vielmehr selbst, und das schreibende Ich ist

5 Ebda.

6 In einem Brief vom 28. April 1841 an seinen Freund Karl August Varnhagen von Ense klingt jedoch noch im Projekt des Kosmos diese autobiographische Dimension unüberhörbar mit: „So sollte das Buch der Reflex meines Selbst, meines Lebens, meiner uralten Person sein.“ Briefe von Alexander von Humboldt an Varnhagen von Ense, S. 91.

7 Vgl. den in seinen Mythologies abgedruckten Text von Barthes, Roland: L'homme-jet. In (ders.): Oeuvres complètes, Bd. 1, S. 619-621. 
seinerseits beständig auf Reisen - so wie Humboldt selbst sich nach seiner Rückkehr aus Amerika und einer sich anschließenden Italienreise nur vorübergehend in Berlin aufhielt, bevor er ab November 1807 für zwei Jahrzehnte Paris zu seinem vielfach durch neuerliche Reisen rhythmisierten Lebensmittelpunkt machte. Das Nomadentum ist für Humboldt keineswegs nur eine Metapher.

Die ruhelose Bewegung auf der Suche nach Neuem, die damit in Verbindung stehende Unabgeschlossenheit der eigenen Texte, die Erfahrung einer intensiven Beschleunigung des Lebens und die Selbstbezüglichkeit des Ichs in variierten autobiographischen Schreibformen bilden wesentliche Grundlagen einer modernen Subjektivität, die ihren Niederschlag nicht nur im ästhetischen, sondern auch im epistemologischen Bereich, in den Grundlagen des Denkens, findet. Stand Georg Forster, wie wir sahen, an einem Anfang des reiseliterarischen Schreibens in der Moderne, so sind wir bei Humboldt bereits auf sicherem Terrain innerhalb der Moderne. Versuchen wir, die Strukturen dieses Schreibens in der Moderne noch genauer zu fassen, um daraus auch Grundlagen für unsere weitere Beschäftigung mit der Reiseliteratur im 19. und 20. Jahrhundert in unserer Vorlesung zu gewinnen.

Ähnlich wie Rousseau in seinen stets wiederholten und abgewandelten Szenen der Illumination, der plötzlichen 'Erleuchtung' hinsichtlich seines späteren Lebensweges, hat auch Alexander von Humboldt immer wieder von neuem die Urszene des Erwachens seiner Lust, ferne Länder zu besuchen, geschildert und literarisch in Szene gesetzt. Schon während seiner amerikanischen Reise verfasste er im August 1801 in Santa Fe de Bogotá, der Hauptstadt des damaligen Neu-Granada, eine autobiographische Skizze, in der er sich darüber klar zu werden versuchte, wie er darauf verfallen war, „entfernte Pläne“ zu schmieden. ${ }^{8}$ In Mes confessions ist es - wie noch in abgewandelter und erweiterter Form im $\operatorname{Kosmos}^{9}$ - der Anblick exotischer Pflanzen, der ihn zu dem Entschluss

8 Dieser auf den 4. August 1801 datierte Text findet sich in der von Kurt-R. Biermann herausgegebenen und bereits angeführten Sammlung (Humboldt, Alexander von: Aus meinem Leben) unter dem Titel „Ich über mich selbst (Mein Weg zum Naturwissenschaftler und Forschungsreisenden 1769-1790)“; dort lesen wir: „Meine Reise mit Forster in das Gebirge von Derbyshire vermehrte jene melancholische Stimmung. Das Dunkel der Casteltoner Höhen verbreitete sich über meine Phantasie. Ich weinte oft, ohne zu wissen warum, und der arme Forster quälte sich zu ergründen, was so dunkel in meiner Seele lag. Mit dieser Stimmung kehrte ich über Paris nach Mainz zurück. Ich hatte entfernte Pläne geschmiedet.“ (S. 40).

9 Vgl. Humboldt, Alexander von: Kosmos, Bd. 2, S. 5: „Kindliche Freude an der Form von Ländern und eingeschlossenen Meeren, wie sie auf Karten dargestellt sind, der Hang nach dem Anblick der südlichen Sternbilder, dessen unser Himmelsgewölbe entbehrt, Abbildungen von Palmen und libanotischen Cedern in einer Bilderbibel können den frühesten Trieb nach Reisen in ferne Länder in die Seele pflanzen. Wäre es mir erlaubt, eigene Erinnerungen anzurufen, 
führt, „Europa zu verlassen““. ${ }^{10}$ „Der Anblick exotischer Pflanzen, selbst in getrocknetem Zustand in Herbarien, erfüllte meine Einbildungskraft mit jenen Freuden, welche die Vegetation klimagünstigerer Länder bietet. "11 Humboldt konnte sich bis ins hohe Alter immer wieder an diesen (selbstgeschaffenen) Szenarien erfreuen.

Der Reise exotischer Pflanzen in die Berliner Herbarien entspricht im weiteren Fortgang eine umgekehrte Bewegungsrichtung, wobei zunächst eine europäische Reise zum Vorspiel der großen außereuropäischen Forschungsreise wird. So kommt es im Frühling jenes Lebens zu einer Reiseerfahrung, die literarisch wie wissenschaftlich zum Bezugspunkt und in der Folge überwundenem, aber nie vergessenem Modell der späteren Expedition werden sollte. Und nicht umsonst bilden die Reisenotizen von dieser Reise den Auftakt der sogenannten Amerikanischen Reisetagebücher.

Au printemps, M. Georges Forster, avec qui j'avais lié connaissance à Mayence, me proposa de le suivre en Angleterre dans ce voyage rapide qu'il a décrit dans un petit ouvrage (Ansichten, etc.) justement célèbre par l'élégance du style. Nous passâmes par Hollande, l'Angleterre et la France. Ce voyage cultivant mon esprit me décida aussi plus que jamais pour le voyage hors d'Europe. Je vis alors la première fois la mer à Ostende, et je me souviens que cette vue fit la plus grand impression sur moi. Je vis moins la mer que les pays auxquels cet élément devait un jour me porter. ${ }^{12}$

Humboldt vervielfacht die Ausgangspunkte für seine ständig fortgesetzte Reisetätigkeit, die gewisslich nicht erst mit Georg Forster begonnen hatte. Aber die Reise mit dem Weltumsegler nach Holland, England und Frankreich - drei ausgeprägte Führungsmächte der Globalisierung der Welt - markierte doch einen tiefen Einschnitt in Humboldts Vorstellungswelt, nicht zuletzt auch aufgrund des 'Besuches' der Französischen Revolution an der Seite des durchaus revolutionären Forster.

Wieder ist es die Ansicht, der Blick und damit der Gesichtssinn, der zum Auslöser des Begehrens wird, Europa zu verlassen und ferne Länder zu besuchen.

mich selbst zu befragen, was einer unvertilgbaren Sehnsucht nach der Tropengegend den ersten Anstoß gab, so müßte ich nennen: Georg Forster's Schilderungen der Südsee-Inseln; Gemälde von Hodges die Ganges-Ufer darstellend, im Hause von Warren Hastings zu London; einen colossalen Drachenbaum in einem alten Thurme des botanischen Gartens bei Berlin.“ Später sollte für ungezählte Reisende - darunter insbesondere den jungen Charles Darwin Humboldts Reisewerk seinerseits zum Auslöser und Modell des eigenen Reisens wie des eigenen Schreibens werden. Reisen macht Lust auf Reisen.

10 Humboldt, Alexander von: Mes confessions, S. 181.

11 Ebda.

12 Ebda., S. 182. 
Man könnte fast von einer concupiscentia oculorum sprechen, die Humboldt ergriff. Lassen wir auch hier das damals etwas unsichere Französisch Alexander von Humboldts - das in der vorliegenden Übersetzung nicht immer geglättet wurde - beiseite, um die angestrebte Bewegungsrichtung der Humboldt'schen Gedanken genauer zu erkennen.

Forster ist dabei für Humboldt wichtig. Denn wie aus der Perspektive des erzählenden Ich die gemeinsame Reise des erzählten Ich mit Georg Forster zur Prä-Figuration der amerikanischen Reise wird, so haftet an dem Reisegefährten die Erfahrung, das Prestige und Renommee einer Umsegelung des gesamten Erdballs. Forsters von Humboldt erwähnte Ansichten vom Niederrhein, von Brabant, Flandern, Holland, England und Frankreich im April, Mai und Junius 1790 verwiesen damit zurück auf jene 1777 in englischer und 1778 bis 1780 in deutscher Fassung vorgelegte Reise um die Welt, mit der wir uns bereits ausführlich beschäftigt haben. Wir haben Georg Forster ja bereits kennengelernt und können verstehen, dass Alexander ihm als ein Forscher nachfolgte, dem es nicht um das Fliegenbeinzählen ging.

Denn der Verfasser des Versuchs über die gereizte Muskel- und Nervenfaser war alles andere als ein eingefleischter Mikrologe, der beim wissenschaftlichen Fliegenbeinzählen stehengeblieben wäre. Er betrachtete den Mikrokosmos stets in seinen wechselseitigen Beziehungen zum Makrokosmos und scheute - als Makrologe, der er auch war - nie davor zurück, über das Große, das Ganze zu sprechen. Ganz im Sinne von Georg Forster, nur mit den avancierteren Mitteln seiner eigenen Zeit, ging es Alexander von Humboldt um alles.

Benannte Forsters Vorrede zum Bericht über die Entdeckungsreise Cooks die methodologischen und epistemologischen Prämissen einer Forschungsreise neuen Typs, so setzte Alexander von Humboldt am Ende des 18. Jahrhunderts diese Konzeption in die Tat um, indem er sie als Forschungsreise im modernen Sinne - und im Sinne der Moderne - anging und durchführte. Humboldt stand in diesem Sinne in der Tradition Forsters. Sein Nomadenleben verband sich auf diese Weise nicht nur mit einem nomadischen Schreiben, sondern auch mit einer nomadisierenden Wissenschaftskonzeption, die an Forsters Vorstellungen geschult weit über diese hinausging und einen neuen Typ des Wissens hervorbrachte, der - so scheint es - erst seit dem Ausgang des 20. Jahrhunderts aus einem neuen, transdisziplinären Wissenschaftsverständnis heraus begriffen werden konnte. Dies macht Alexander von Humboldt zu unserem Zeitgenossen.

Vor diesem Hintergrund wird verständlich, dass Humboldt in seinen 'Bekenntnissen' großen Wert darauf legte, nicht nur durch die von ihm herbeigeschleppten Dinge und Fakten, sondern auch (und mehr noch) durch jene Ideen zu wirken, die er in anderen entstehen ließ. Dies deutet auf ein höchst modernes Wissenschaftsverständnis. Alexander von Humboldt begriff sich ohne 
jeden Zweifel als Teil eines Netzwerks der Wissenschaft, das seit der zweiten Hälfte des 18. Jahrhunderts im Kontext des von Jürgen Habermas beschworenen 'Projekts der Moderne' seine Netze - nicht nur die der Längen- und Breitengrade - über den gesamten Globus ausgeworfen hatte. Im Kern dieses Wissenschaftsverständnisses standen der Austausch, die Zirkulation von Wissen, die Zusammenarbeit über die Grenzen von Nationen, vor allem aber auch über die Grenzen der sich immer weiter ausdifferenzierenden Disziplinen hinweg.

Der Bescheidenheitstopos verbirgt freilich nicht, dass es Alexander von Humboldt gerade auf die Verbreitung neuer Ideen ankam, die ihrerseits auf einer Verbindung der Sammeltätigkeit mit dem Versuch beruhte, das Gesammelte in einer Gesamtsicht zu einem Ganzen zusammenzudenken. Darum wird ihm das Meer, das er gleichsam unter den Augen des weitgereisten Georg Forster bei Ostende zum ersten Male erblickte, auch nicht zum trennenden, sondern alles in eine weltumspannende Kommunikation einbindenden, alles miteinander verbindenden Element. Diese Sichtweise des Meeres ist für die Humboldt'sche Wissenschaft charakteristisch und weist auf die enorme Bedeutung der Zirkulation für den Preußen hin. Auch auf diesem Feld ist alles bei Humboldt in Bewegung.

Auf diese Weise wurde die Reise zur Voraussetzung eines weltschaffenden, globalen Denkens, das nicht auf Sammeln, sondern Verbinden angelegt war. Und nach eben dieser Logik musste die amerikanische durch eine komplementäre asiatische Forschungsreise ergänzt und vervollständigt werden. Diese (stets prekäre) Vervollständigung aber konnte sich nur auf der Grundlage moderner Subjektivität ergeben, unter Einbeziehung eben jener perfectibilité, die Rousseau als Charakteristikum des Menschen beschrieben hatte und die für Humboldt zu jenem movens im Zeichen moralischer Unruhe wurde, das nicht nur das Denken, sondern auch den Denker selbst, nicht nur die Literatur, sondern auch den Literaten selbst in Bewegung setzte. Humboldt wusste, dass die Voraussetzung für ein Schreiben über den gesamten Erdball eine zweite, von ihm selbst durchzuführende transkontinentale Reise sein musste.

Noch ein Wort zum verbindenden Element par excellence. Das alles miteinander in Verbindung setzende Meer wird nicht nur zu einem Forschungsobjekt Alexander von Humboldts, sondern zu einem vieldeutigen Symbol, das ebenso den Weg des Reisenden bereiten als auch dessen Untergang herbeiführen kann. An zahlreichen Stellen der Relation historique und anderer Schriften erscheint das Meer überdies als Refelxionsfläche des Sternenhimmels - mithin des Kosmos und führt dem Menschen die Gesamtheit der Schöpfung wie ein Spiegel vor Augen. Die im Kontext von Humboldts kosmologischer Metaphorik beobachtbare Ambivalenz des Wassers als lebensspendende und zerstörerische, die Kräfte des Menschen weit übersteigende Macht besitzt nicht nur in Hinblick auf die wissenschaftliche, sondern auch auf die literarische Gestaltung des Reiseberichts größte 
Bedeutung.. Gerade die Beschreibungen eines drohenden Schiffbruchs, eines bevorstehenden Untergangs nehmen - auch in den Amerikanischen Reisetagebüchern - bei Humboldt einen außerordentlich bedeutungsvollen Raum ein.

Das Meer setzt alles miteinander in Verbindung und Wechselwirkung. Setzen die Meeresströmungen verschiedenste Weltgegenden miteinander in Kontakt, so bringt auch der Mensch mit Hilfe seiner Schiffe die unterschiedlichsten Gesellschaften und Kulturen in eine nicht nur ökonomische, am modernen Welthandel ausgerichtete, sondern auch am interkulturellen Austausch interessierte Beziehung. Verwunderlich ist es daher nicht, dass Alexander von Humboldt stets größte Sorgfalt auf jene Augenblicke der Landung oder der Abreise legte, die man - wie wir sahen - als reiseliterarische Orte höchster Signifikanz ansehen muss.

Das Humboldt'sche Reisewerk bietet eine Fülle derartiger Darstellungen; doch dürfen wir uns der Wahl des kubanischen Schriftstellers und poeta doctus Alejo Carpentier anschließen, der für den Auftakt von La ciudad de las columnas, der literarischen Liebeserklärung an seinen (vorgeblichen) Geburtsort Havanna, eine Passage aus dem 28. Kapitel der Reise in die Äquinoktial-Gegenden des Neuen Kontinents wählte, welches später als Politischer Versuch über die Insel Cuba eigenständig in Buchform erschien.

Diese Passage inszeniert die Ankunft des Europäers im Hafen von Havanna, den Alexander von Humboldt ein erstes Mal am 19. Dezember 1800 vom Meer aus erblickte und für uns aus einer wunderbar mobilen Perspektive evoziert:

Bei der Einfahrt in den Hafen ist der Anblick von Havanna einer der strahlendsten und malerischsten unter all jenen, die man an den Gestaden des äquinoktialen Amerika nördlich des Äquators genießen kann. Dieser von den Reisenden aller Nationen gefeierte Ort besitzt weder die Überfülle an Vegetation, welche die Ufer des Flusses von Guayaquil schmückt, noch die wilde Majestät der felsigen Küste von Rio de Janeiro, zwei Häfen der südlichen Hemisphäre: Doch die Anmut, die in unseren Klimaten die Szenerie einer in Kultur genommenen Natur verschönert, mischt sich hier mit der Majestät der Pflanzenformen, mit der organischen Kraft, welche die torride Zone charakterisiert. In einer Mischung von so süßen Eindrücken vergißt der Europäer die Gefahr, die ihn im Schoße der volkreichen Städte der Antillen bedroht; er sucht, die verschiedenartigen Elemente einer weiten Landschaft zu ergreifen, diese Befestigungen zu betrachten, welche die Felsen im Osten des Hafens krönen, dieses innere Becken, das von Dörfern und Höfen gesäumt ist, diese Palmen, die sich in schwindelnde Höhe erheben, diese Stadt, die halb von einem Wald an Schiffsmasten und den Segeln der Schiffe verborgen wird. ${ }^{13}$

13 Humboldt, Alexander von: Relation historique du Voyage aux Régions équinoxiales du Nouveau Continent ... Nachdruck des 1814-1825 in Paris erschienenen vollständigen Originals, besorgt, eingeleitet und um ein Register vermehrt von Hanno Beck. Bd. III. Stuttgart: Brockhaus 1970, S. 348. 


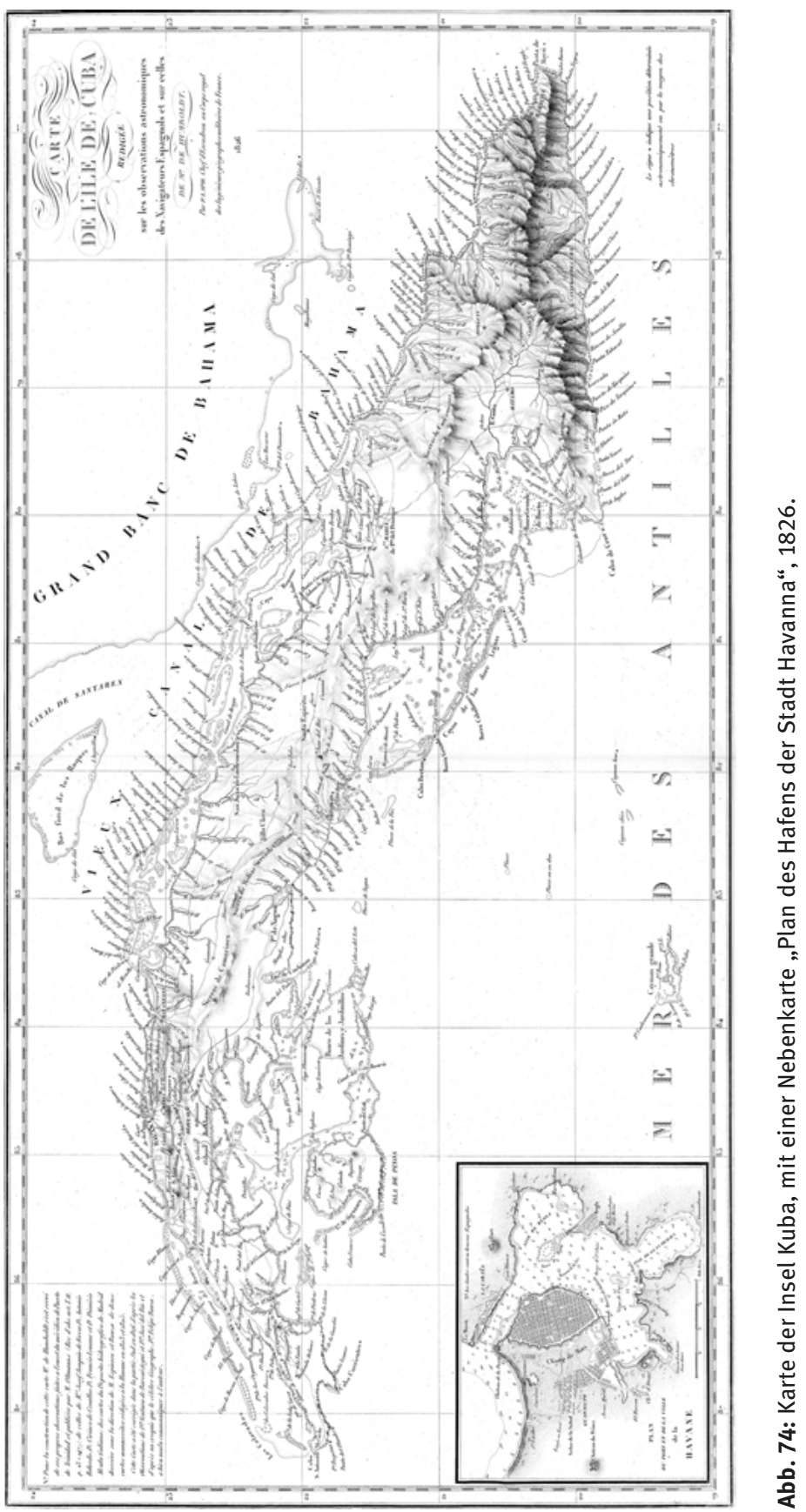


Wir sehen förmlich Humboldt in den Hafen von Havanna gleiten. In dieser kurzen, literarisch sorgfältig gestalteten und zunächst im Zeichen des Pittoresken modellierten Passage wird der Anblick bei der Einfahrt in den kubanischen Haupthafen sogleich mit jenem anderer Häfen der amerikanischen Tropenländer in Beziehung gesetzt, wobei die Erzählerinstanz zum einen auf den Hafen von Guayaquil (den Alexander von Humboldt aus eigener Erfahrung kannte) und auf jenen von Rio de Janeiro (den er selbst niemals besuchte) vergleichend verweist. Dies macht auf die transareale Perspektivik der gesamten Humboldt'schen Sichtweise aufmerksam.

Die präsentische Darstellung bedeutet nicht, dass wir uns auf der Ebene des erzählten (beziehungsweise des reisenden) Ichs befinden; die Rücknahme der im vorangehenden Abschnitt noch dominanten individualisierten Perspektive in ein verallgemeinerndes 'man' erschließt sich jedoch nur einer genaueren Analyse als Signal, dass die Beobachterposition deutlich gewechselt hat. In der Tat könnte ein unaufmerksamer Leser zu dem Schluss kommen, Humboldt habe den Hafen von Rio de Janeiro aus eigener Kenntnis beschrieben - ein Beispiel für jene vom Text quasi suggerierte Annahmen, wie sie sich immer wieder selbst in der Humboldt-Forschung finden. Doch Humboldt kannte den brasilianischen Hafen nur aus bildlichen Darstellungen und Reisebeschreibungen. Tatsächlich beruht der Vergleich, der hier in die Darstellung einfließt, nur zum Teil auf eigenen Erfahrungen des Reisenden, wenngleich er auch in dieser Form ein diskursives Merkmal des Humboldt'schen Schreibens darstellt, das sich stets in weltumspannenden Vergleichen bewegt. Dies ist ein zentrales Merkmal der écriture des preußischen Natur- und Kulturforschers.

Beachten wir dabei aber trotz aller Betonung von Augenzeugenschaft: Das komparative, transareale Element als eine der epistemologischen Grundlagen der nomadisierenden Schreibweise Humboldts ist nicht notwendig an persönliche Beobachtung und Erfahrung gekoppelt, denn der Anblick der Häfen von Guayaquil und Rio de Janeiro wird auf derselben Ebene des 'Wahrheitsanspruches' behandelt. Wir haben es in dieser auf den ersten Blick unscheinbaren Passage weder mit dem erzählten noch mit dem erzählenden Ich zu tun, sondern mit einer Erzählinstanz, die gleichsam von höherer Warte aus die Einzelphänomene überblickt und einordnet. Diese narrative Instanz ist für Humboldts Schreiben von großem Gewicht. Da diese wissenschaftliche Erzählinstanz ebenfalls in der ersten Person Singular auftritt, können wir sie auch als das wissenschaftliche Ich - das bisweilen beide Zeitebenen miteinander verbindet - bezeichnen. Sie geht in die Konstituierung des wissenschaftlichen Subjekts in grundlegender Weise ein. Ihr obliegt es in diesem Falle, die Erfahrung anderer Reisender vergleichend einzublenden, wenn auch in einer sehr ambivalenten Art und Weise, die nur dem mit Humboldts gesamter Reiseroute vertrauten Leser erlaubt, die 
individuelle von der intertextuellen, also aus anderen Texten anderer Autoren bezogene Erfahrung zu unterscheiden.

Zugleich macht diese Passage deutlich, dass auch viele andere Reisende aus aller Herren Länder diesen Hafen besucht haben, so dass Bewegung und Transportmittel, aber auch Fremderfahrung und (eine mitunter auch bedrohliche, spannungserzeugende) Alterität ins Zentrum des Interesses des (Be-)Schreibenden wie seiner Leserschaft rücken. Der angesprochene (explizite) Leser gehört ebenso wie der Reisende einer anderen Klimazone an, deren Reize einer „kultivierten Natur“ sich hier mit tropischen Vegetationsformen verbinden. Angesichts der Mischung der durch diese Reize unterschiedlicher Herkunft ausgelösten Eindrücke vergesse der Europäer, dessen Perspektive die angeführte Passage unübersehbar beherrscht, jene Gefahren, welche die Andersheit des kulturellen Raumes jener „bevölkerungsreichen Städte der Antillen“ (auch dies wieder ein impliziter Vergleich, der nur zum Teil auf die eigene Erfahrung Humboldts zurückgeführt werden kann) bereithält.

Dieser Blick des Europäers aber versucht nun, die verschiedenartigen Elemente $\mathrm{zu}$ einer weiten Landschaft ${ }^{14}$ - und damit $\mathrm{zu}$ einer abgrenzbaren Einheit und Vorstellung - zusammenzufügen. Die Addition der einzelnen Elemente gerät nicht zu einer simplen Sammlung, sondern fügt sich zu einer (ästhetisch gestalteten) Gesamtheit, die aus den einzelnen Details gleichsam als Grundidee destilliert wird. Humboldt ordnet die Bestandteile zum einen nach dem Grade ihrer unmittelbaren Sichtbarkeit - und damit vom beweglichen Beobachterstandpunkt des in den Hafen einfahrenden Reisenden - her (von der Höhe der festungsgekrönten Felsen bis hinab zur halb verborgenen Stadt) und zum anderen gemäß ihrer dynamischen Beziehung zu den Bereichen von Kultur und Natur. Er geht dabei als versierter Schriftsteller planmäßig und geordnet vor.

So wird das vertikale, landschaftsbestimmende Element der Palmen, die nicht nur bis heute das Symbol der Insel geblieben sind, sondern auch für Humboldt zur Chiffre tropischer Vegetation und Lebenskraft wurden, überführt in einen „Mastenwald“, so dass der Bereich der Natur in jenen der Kultur transponiert und 'übersetzt' wird. Er folgt darin Georg Forster, der in dem der Stadt Amsterdam und ihrem Hafen gewidmeten Kapitel seiner Ansichten vom Niederrhein ebenfalls mit dem Natur und Kultur verbindenden Wald von Masten sowohl der Kriegs- als auch der Handelsschiffe spielt. Der Übergang zwischen

14 Zur Fassung der Natur als Landschaft ausgehend von Petrarcas Aufstieg zum Mont Ventoux vgl. Ritter, Joachim: Landschaft. Zur Funktion des Ästhetischen in der modernen Gesellschaft. In (ders., Hg.): Subjektivität. Sechs Aufsätze. Frankfurt am Main: Suhrkamp 1989, S. 141-163. Zur Bedeutung der Konfiguration von Landschaft zu einer Landschaft der Theorie vgl. Ette, Ottmar: Roland Barthes. Landschaften der Theorie. Konstanz: Konstanz University Press 2013. 
Natur und Stadt wird just von jenen Schiffen bewerkstelligt, die als mobile Transportmittel jene Bewegung sicherstellen, die den europäischen Reisenden in die Tropen führt und ihn auch wieder zurück in seine Heimat bringen wird. Alles steht folglich miteinander in bewegter Wechselwirkung.

Die Spannung zwischen Ich und Landschaft, die in der europäischen Reiseliteratur die Grundlage für die Konstituierung moderner Subjektivität bildet, wird in dieser Passage durch eine Spannung ergänzt, die sich zwischen der Beobachterperspektive des erzählten Ich (des Reisenden) und der höhergelagerten Instanz des wissenschaftlichen Ich aufbaut. Humboldt geht hier als literarischer Autor sehr feingliedrig vor. Im Zwischenraum, der durch diese beiden Pole gekennzeichnet wird, situiert sich ein Gutteil des Humboldt'schen Schreibens, dessen einzelne Instanzen in der Folge noch weiter analysiert und differenziert werden sollen.

Als konstitutiv für dieses Schreiben aber lässt sich schon jetzt der graduelle Übergang und die Verzahnung von Natur und Kultur, der wechselnde Beobachterstandpunkt mit einer jeweils veränderten, in Bewegung befindlichen Beobachterperspektive und die Auseinandersetzung mit anderen Texten und Reiseberichten erkennen, wobei diese Elemente Eingang in eine sorgfältige literarische Strukturierung finden, die ihrerseits in der Markierung eines reiseliterarischen Ortes Funktionen für den Humboldt'schen Text in seiner Gesamtheit übernimmt. Humboldts Schreiben ist mit seiner Theorie von Natur, Kultur und Wissenschaft sehr eng verwoben. Alles unterliegt dabei einer ständigen Bewegung, so dass in Humboldts Schriften oftmals eher von einer Strukturierung als von einer festen, ein für alle Mal fixierten Struktur gesprochen werden kann. Das Mobile dominiert.

Aber da gibt es noch einige wesentliche reiseliterarische Techniken und Kniffe mehr. Denn auch die philosophischen Rahmungen, ${ }^{15}$ die den Reisebericht häufig zu Beginn und am Ende der einzelnen Kapitel prägen, sind Textelemente, die weniger einen statischen Rahmen darstellen als eine Abfolge bedeutungsstrukturierender diskursiver Elemente bilden. Mit ihrer Hilfe wird das bei oberflächlicher Betrachtung Disperse - wie im Blick des Europäers über die Umgebung des Hafenbeckens von Havanna - in ein einheitliches, wenn auch vielgestaltiges Ganzes überführt. Die vereinzelten Elemente verlieren gleichsam

15 Im Nachwort zu meiner Ausgabe von Alexander von Humboldts Reise in die ÄquinoktialGegenden des Neuen Kontinents (S. 1563-1597) habe ich auf diese literarische Technik aufmerksam gemacht. Vgl. Humboldt, Alexander von: Reise in die Äquinoktial-Gegenden des Neuen Kontinents. Herausgegeben von Ottmar Ette. Mit Anmerkungen zum Text, einem Nachwort und zahlreichen zeitgenössischen Abbildungen sowie einem farbigen Bildteil. 2 Bde. Frankfurt am Main - Leipzig: Insel Verlag 1999. 
ihren autonomen Status und werden in ein Gesamtbild integriert, das jedem Element seinen Sinn verleiht.

Den bedeutungsstrukturierenden Einheiten kommt dabei weniger eine definitorische (also fixierende, fest-stellende) als eine kommunikative Funktion zu, welche die Kollektion der gesammelten Elemente in ein dynamisches Netzwerk verwandelt. Auch hier geht es um eine Zirkulation der Elemente, um eine Zirkulation des Wissens. Dadurch stellen die Humboldt'schen Texte jene übergreifenden Kodes zur Verfügung, kraft derer die verschiedensten Einzelerscheinungen dekodiert und in ihrem Funktionszusammenhang innerhalb des 'Naturgemäldes' eingeordnet und verarbeitet werden können. Das Naturgemälde wird nicht nur entworfen, es liefert die Codes zu seiner Entschlüsselung gleich mit. Dies fördert die Zugänglichkeit des Humboldt'schen Reiseschreibens wie auch der Humboldt'schen Wissenschaft ganz allgemein. Die Verbindung zwischen Reisen und Schreiben bleibt transparent.

In seinen erstmals im Jahre 1808 erschienenen Ansichten der Natur, die paratextuell schon in ihrem Titel auf die Vorherrschaft des Visuellen und die Pluralität der Standpunkte aufmerksam machen, intertextuell den Bezug zu Forsters Ansichten vom Niederrhein herstellen und rezeptionsgeschichtlich Humboldt bei einem breiteren deutschsprachigen Publikum bekannt machten, findet sich nach der Vorrede gleich zu Beginn des Bandes ein Text, der uns die Analyse grundlegender Schreibstrategien des preußischen Forschungsreisenden ermöglicht. An ihm wollen wir die verschiedenen Elemente der Humboldt'schen écriture, des Humboldt'schen Reiseschreibens, noch genauer studieren.

'Ueber die Steppen und Wüsten' setzt mit der Erwähnung eines „hohen Granitrückens“ ein, der geologisch dem „Jugendalter unseres Planeten“ zugeordnet wird. ${ }^{16}$ Von hier aus schweift der Blick des Betrachters über eine Landschaft, deren Horizont und Begrenzung „in schwindender Ferne“ erscheint. $^{17}$ Es handelt sich, wie wir gleich sehen werden, um eine Landschaft der Theorie: um eine Landschaft, an deren Exemplum Humboldt seine theoretischen Überlegungen, aber auch seine philosophischen Projektionen vorführen will.

Damit wird bereits im ersten Abschnitt nicht nur die im Buchtitel hervorgehobene Visualisierung, sondern auch die im Titel mitbedeutete erhöhte Beobachterposition von 'Ueber die Steppen und Wüsten' eingelöst und mit einer individuellen Perspektive verknüpft. Es ist die Perspektive des reisenden Ich. Dieser Blickwinkel wird dann zu Beginn des zweiten Abschnitts in Bewegung

16 Humboldt, Alexander von: Ueber die Steppen und Wüsten. In (ders.): Ansichten der Natur mit wissenschaftlichen Erläuterungen. Bd. 1. Tübingen: Cotta 1808, S. 1.

17 Ebda., S. 2. 
gesetzt, tritt doch „der Wanderer betroffen an den öden Rand einer pflanzenleeren Wüste“, die in starkem Kontrast zur „üppigen Fülle des organischen Lebens“ steht, ${ }^{18}$ deren Bereich er soeben verlässt. Die sich daraus ergebende Rahmung der Llanos durch eine tropische Vegetation verweist auf den Schlussteil des Textes, in welchem eben diese „Fülle“ wiederum in den Blick rückt und sich damit eine Kreisstruktur abzeichnet, die dem Text auch in Bezug auf die Spannung zwischen organischer und anorganischer Natur zugrunde liegt. Es sind Spannungsverhältnisse, die nicht statischer Natur sind, sondern zugleich auch auf eine Wechselwirkung abzielen.

Das Grundschema des literarisch-wissenschaftlichen Essays entfaltet sich in einer Diachronie, die mit dem Urgestein im „Jugendalter“ einsetzt, wobei die „Bilder der Vorzeit“ noch gegenwärtig durch „nächtliche Täuschung“ - also im Beobachtersubjekt - zurückgerufen und gleichsam vor Augen geführt werden. ${ }^{19}$ Vor dem Auge des Betrachters ersteht das neptunistisch eingefärbte Bild eines in geologischen Zeiträumen ablaufenden Sedimentationsprozesses, indem an die Stelle der wasserarmen Wüste die Meeresfläche des Ozeans tritt und damit gleichsam eine Landschaft hervorbringt, die Ausfluss einer Theorie ihrer Entstehung und der Entstehung des ganzen Planeten ist:

Denn wenn im raschen Aufsteigen und Niedersinken die leitenden Gestirne den Saum der Ebene erleuchten; oder wenn sie zitternd ihr Bild verdoppeln, in der untern Schicht der wogenden Dünste, glaubt man den küstenlosen Ozean vor sich zu sehen. Wie dieser erfüllt die Steppe das Gemüth mit dem Gefühl der Unendlichkeit. Aber freundlich zugleich ist der Anblick des klaren Meeresspiegels, in dem sich die leichtbewegliche sanft aufschäumende Welle kräuselt. Todt und starr liegt die Steppe hingestreckt, wie die nackte Felsrinde eines verödeten Planeten. ${ }^{20}$

Humboldt führt uns gleichsam in eine Ur-Landschaft, die uns mit den alles beherrschenden Naturkräften vertraut macht. Die Überblendtechnik resultiert keineswegs in einer metaphorischen Verschmelzung von Steppe und Meer, auch wenn beide Räume in ihrer Unendlichkeit den Reisenden, der sie durchquert, in ihren Bann ziehen und seine Gedanken und Vorstellungen fesseln. Aufschlussreich ist überdies die Gestaltung des impliziten literarischen Raumes, also jenes Horizonts von Bezügen $\mathrm{zu}$ anderen Texten, die nicht explizit im Text benannt werden. Denn kaum verhüllt (wenn auch bislang noch nicht erkannt) sind die Anspielungen auf die Genesis: Gottes Scheidung zwischen

18 Ebda. Humboldt verstärkte diesen Kontrast in späteren Ausgaben des Textes, indem er nun von einer „baumlosen, pflanzenarmen Wüste“ sprach.

19 Ebda.

20 Humboldt, Alexander von: Ueber die Steppen und Wüsten, S. 2f. 
den Wassern oberhalb und unterhalb des Himmelsgewölbes am ersten und die Trennung von Erde und Meer am zweiten Tag der Schöpfung: „Da sprach Gott: 'Die Erde lasse Grünes hervorsprießen, samentragende Pflanzen sowie Fruchtbäume, die Früchte bringen nach ihrer Art, in denen Samen ist auf Erden!' Und es geschah so.“21 Humboldt liefert uns eine Entstehung der Welt, vielleicht sogar eine Schöpfung der Erde, aber ohne jeden Gott.

So unterliegt der durch eine optische Täuschung hervorgerufenen 'Ansicht' der Erdentstehung nicht nur ein erdgeschichtlich-naturwissenschaftlicher, sondern auch ein christlich-transzendenter Bezugstext, der in der Folge innerhalb eines profanen Settings in impliziter Intertextualität weiterentwickelt wird. Die zunächst verdoppelte Beobachterperspektive des Wanderers und des auktorial gestalteten Erzählers wird durch eine ebenfalls in der dritten Person agierende wissenschaftliche Instanz ergänzt, die - wie die Figur des wissenschaftlichen Ichs im Reisebericht - die diskursiven Passagen dominiert und an dieser Stelle zunächst den weltweiten transarealen Vergleich mit anderen Regionen unseres Planeten einleitet. Innerhalb dieses durch Narrativierungen immer wieder aufgelockerten weltweiten Vergleichs rücken ebenso die Vegetation als auch die Menschheitsentwicklung in den Blick, so dass sich die Erde vor den Augen des Lesers in einer Art entsakralisierter Schöpfungsgeschichte zu bevölkern beginnt. Alexander von Humboldts Welt bedarf keines Schöpfergottes, sie kommt gut ohne ihn aus, ohne dass sich ihr Verfasser doch hinsichtlich der Transzendenz positioniert hätte.

Dieser Prozess aber wird in der Rückkehr zu den 'Steppen und Wüsten' von Tierra firme (des heutigen Venezuela) zurückgedreht:

Das Interesse, welches dies Gemälde dem Beobachter gewähren kann, ist ein reines Naturinteresse. Keine Oase erinnert hier an frühe Bewohner, kein behauener Stein, kein verwildeter Fruchtbaum an den Fleiß untergegangener Geschlechter. Wie den Schicksalen der Menschen fremd, allein an die Gegenwart fesselnd, liegt dieser Erdwinkel da, ein wilder Schauplatz des freien Thier- und Pflanzenlebens. ${ }^{22}$

Diese Landschaft der Theorie ist vom Menschen nicht besiedelt, ja ist absolut menschenfrei. So erscheint nun auf der diachronen Achse, an die Trennung von Meer und Erde und die Gebirgsbildung anschließend, eine Welt der Natur in der Freiheit von Flora und Fauna, die entgegen der Schöpfungsgeschichte dem Menschen nicht untertan ist, ja den Menschen noch nicht einmal kennt. Einige Jahre

21 Die Heilige Schrift des Alten und Neuen Testamentes. Nach den Grundtexten übersetzt und herausgegeben von Vinzenz Hamp, Meinrad Stenzel und Josef Kürzinger. Aschaffenburg: Pattloch ${ }^{19} 1969$ (Genesis 1, 11).

22 Humboldt, Alexander von: Ueber die Steppen und Wüsten, S. 10 f. 
später, in seiner 'Introduction' in die Relation historique hat Humboldt auf ein 'Ungleichgewicht' zwischen Natur und Kultur in Amerika aufmerksam gemacht: „In der alten Welt sind es die Völker und die Abstufungen ihrer Zivilisation, die dem Gemälde seinen Hauptcharakter geben; in der neuen hingegen verschwindet gleichsam der Mensch mit seinen Produkten inmitten einer wilden und gigantischen Natur.“.23

Humboldt versuchte, mit Blick auf seine Leserschaft die reiseliterarischen Konsequenzen für die von ihm angewandten Darstellungstechniken zu ziehen. Auf den folgenden Seiten beginnt er, die einzelnen Elemente dieses Gemäldes nebst den sie bestimmenden Faktoren wie Bodenbeschaffenheit, Klima oder Atmosphäre zu entfalten, um dann - und hier betritt der Mensch dann doch wieder die Bühne - die Entstehung und Bedingungen der Viehzucht in den Steppen des späteren Venezuela vor dem Hintergrund unterschiedlicher kultureller Voraussetzungen zu erörtern. Der Mensch ist für Humboldt ein zentrales Element der Natur: Wie hätte er in seinem Tableau also vollständig fehlen können?

Zunächst betritt der Indio, die indigene Bevölkerung, die Humboldt'sche Landschaft der Theorie. An die Darstellung indianischer Völker der Llanos schließt sich eine Diskussion der Folgen der Entdeckung und Eroberung dieser weiten Landstriche durch die Europäer an, ein geschichtlicher Einschnitt, der in seiner Bedeutung auch für Pflanzen- und Tierwelt herausgearbeitet wird. Denn mit dem ersten Auftauchen von Europäern wird von diesen gleichsam alles in Zirkulation und Bewegung gesetzt.

Erneut werden die diskursiven Teile stets durch die Einschaltung narrativer Passagen rhythmisiert, wobei insbesondere „der wunderbare Kampf der Pferde und Fische“, ${ }^{24}$ der Fang der Gymnoten, Elemente des Kampfes und der Spannung in den Text einbringt (und von jeher die Aufmerksamkeit des Publikums auf sich gezogen hat). Humboldt wusste, wie er für das Lesepublikum seine Landschaft beleben konnte. Eine abschließende philosophische Bemerkung, die thematisch wie syntaktisch noch einmal alles 'Gesammelte' zusammenzuführen und zu einer höheren Einheit zu verbinden sucht, scheint diese Seiten zu rahmen: „alles, wie die Farbe des getheilten Lichtstrals, fließt aus einer Quelle; alles schmilzt in eine ewige allverbreitete Kraft zusammen. “25 Damit ist die Instanz der philosophischen Rahmung aufgerufen.

23 Humboldt, Alexander von: Reise in die Äquinoktial-Gegenden des Neuen Kontinents, Bd. I, S. $35 \mathrm{f}$.

24 Humboldt, Alexander von: Ueber die Steppen und Wüsten, S. 40.

25 Ebda.; in der dritten Auflage seiner Ansichten hat Humboldt diese Passage noch stärker akzentuiert. 
In der Tat tritt nun die auktorial gestaltete Erzählinstanz, nun aber unvermittelt in die erste Person Singular verwandelt und damit eine Identifizierung mit dem realen, also textexternen Autor nahelegend, in den Vordergrund: „Ich könnte hier diesen gewagten Versuch eines Naturgemäldes der Steppe schließen. “26 Doch soll - so die Ausführungen dieser Figur - der Blick auf den letzten Seiten des Textes noch einmal über die Landschaftsgrenzen hinaus erweitert werden. Eine letzte Ausweitung dieses reiseliterarischen Textes steht noch bevor.

Denn der Begriff des 'Naturgemäldes' kündigt bei Humboldt immer das Modell einer Ganzheit an, die stets sowohl wissenschaftlich als auch künstlerischästhetisch gestaltet wird. Der „flüchtige Blick“ über die Grenzen der Llanos erweist sich als Blick auf die Grenzziehungen zwischen Natur und Kultur und die Begrenztheit kultureller Entwicklung überhaupt. Wie im Zeitraffer werden geologische und naturräumliche Verhältnisse, Vegetation und Tierwelt sowie die verschiedenen indianischen Völker ${ }^{27}$ in ihrer Entwicklung aufgerufen, um in den beiden abschließenden Abschnitten des Textes von der Darstellung des Einzelnen zur Darstellung des Ganzen überzugehen. Die Übergänge von den Naturphänomenen zum (der Natur für Humboldt stets zugehörigen) Menschen sind dabei graduell und keineswegs von Fortschrittseuphorie geprägt:

So bereitet der Mensch auf der untersten Stufe thierischer Rohheit, so im Scheinglanze seiner höheren Bildung, sich stets ein mühevolles Leben. So verfolgt den Wanderer über den weiten Erdkreis, über Meer und Land, wie den Geschichtsforscher durch alle Jahrhunderte, das einförmige, trostlose Bild des entzweiten Geschlechts.

Darum versenkt, wer im ungeschlichteten Zwist der Völker nach geistiger Ruhe strebt, gern den Blick in das stille Leben der Pflanzen, und in der heiligen Naturkraft inneres Wirken; oder hingegeben dem angestammten Triebe, der seit Jahrtausenden der Menschen Brust durchglüht, blickt er ahndungsvoll aufwärts zu den hohen Gestirnen, welche in ungestörtem Einklang die alte ewige Bahn vollenden. ${ }^{28}$

Wie bei Dante, aber auch wie bei Immanuel Kant, ist es der Blick in die Sterne, in den 'gestirnten Himmel' des Kosmos, der als Blick in eine Ewigkeit für Sicherheit

26 Ebda.

27 Der Übergang zwischen Tierwelt und Indianern wird im drittletzten Abschnitt vorgenommen und führt dem Leser eine Abfolge vor Augen, die aus heutiger Sicht als höchst problematisch erscheint. So folgen auf die „Tiger und Crocodile“ (also die wilden Tiere) die Pferde und Rinder (mithin die domestizierten, teilweise aber wieder 'verwilderten' Tiere), denen ihrerseits indianische Völkerstämme folgen, die mit „unnatürlicher Begier“ das Blut ihrer Feinde trinken; am Ende dieser Kette stehen wieder andere Indianer, die nur „scheinbar waffenlos“, tatsächlich aber „zum Morde vorbereitet“ sind (ebda., S. 44f). Die indianischen Völker am Orinoco werden somit in eine Abfolge integriert, die sie zu - den Tieren noch nahen - Vermittlern jenes beständigen Kampfes bis in die Stufen der „höheren Bildung“ macht.

28 Humboldt, Alexander von: Ueber die Steppen und Wüsten, S. 45 f. 
und Transzendenz steht. In dieser abschließenden philosophischen Reflexion wird der Wanderer durch den Raum (mithin durch die ersten drei Dimensionen unserer in der theoretischen Grundierung der Vorlesung entworfenen Kartierung), der sich ihm in seiner naturäumlichen Ausstattung darbot, zum Wanderer durch die Zeit, jener vierten Dimension, die ihm ihrerseits in geologischen wie in historischen Größenordnungen entgegentritt. Der durch die verschiedenen Stufen der Zivilisation aufgespannte Raum der Geschichte des Menschen als Geschichte des Kampfes und der Ausrottung - nicht umsonst ist das Buch in Zeiten einer äußersten Bedrohung des preußischen Staatswesens im Vorwort den „Bedrängten Gemüthern“ gewidmet $^{29}$ - wird am Ende von 'Ueber die Steppen und Wüsten' wieder verlassen, um zur anorganischen Natur des incipit, des Textbeginns zurückzukehren. Deutlicher als die deutsche formuliert dies die französische Ausgabe der Tableaux de la Nature, wenn sie diesen Raum sich vom „plus bas degré de la sauvagerie animale“ bis zum „sommet de la civilisation“ erstrecken lässt und damit die distanzierende Metaphorik des 'Scheinglanzes' nicht übernimmt. ${ }^{30}$

Die nicht mit dem realen, textexternen Autor Alexander von Humboldt zu verwechselnde Figur des einsamen Wanderers, die in keiner Weise der historischen Reisegruppe um Alexander von Humboldt und Aimé Bonpland entspricht, tritt an die Stelle der Humboldt'schen Karawanserei und beseitigt aus ästhetischen (und wohl nicht aus narzisstischen) Gründen die Reisegefährten, um immer wieder eine Fokalisierung auf eine einzige Position subjektiver Wahrnehmung sicherzustellen. Zweifellos kommt dieser Figur auch gegenüber dem Lesepublikum eine identifikatorische, die Identifizierung der Leser mit dem Ich fördernde Funktion zu. Die von Humboldt gewählte literarische Technik mag neben einer Vielzahl weiterer Gründe dazu beigetragen haben, dass Aimé Bonpland beim deutschsprachigen Publikum in zunehmendem Maße 'verschwand', obwohl er als Ko-Autor etwa der Relation historique - die freilich Humboldt redigierte - in der Titelei sehr wohl vermerkt war.

Die moderne Subjektivität, die sich aus der rastlosen Bewegung des Wanderers durch die Räume und die Zeiten entfaltet, tritt hier aus der Erfahrung einer durch den Menschen historisch beschleunigten Entwicklung heraus und wird in der Bewegung des Kosmos, etymologisch als Ordnung und Schönheit, im doppelten Wortsinne aufgehoben. Der Wanderer ist eine literarische Figur, welche die Inszenierung einer mobilen Perspektive des modernen Subjekts wirkungsvoll zu verkörpern sucht. Und dieses Subjekt ist ein Subjekt der Moderne.

29 Humboldt, Alexander von: Ueber Steppen und Wüsten, S. VII.

30 Vgl. Humboldt, Alexander von: Tableaux de la Nature. Bd. I. Nanterre: Editions Européennes Erasme 1990, S. 56. 


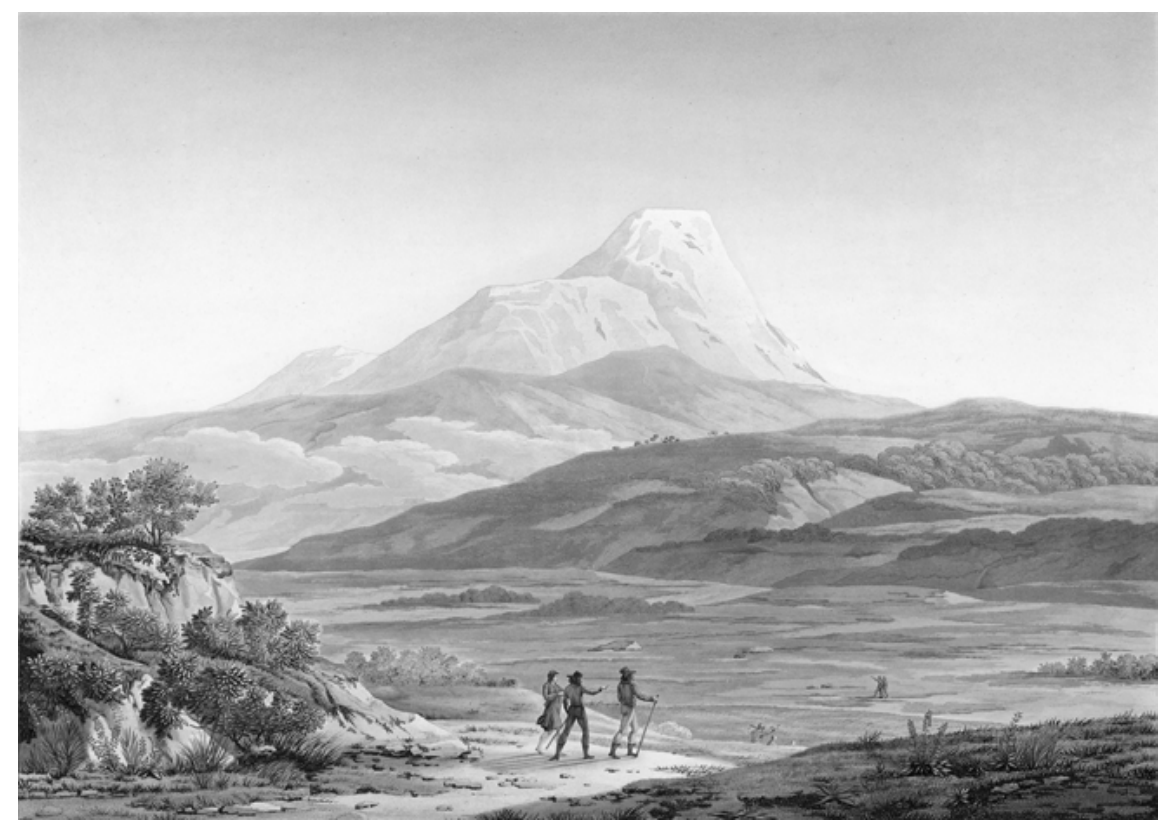

Abb. 75: „Vue du Cayambe“ von Alexander von Humboldt.

Der Blick nach oben in die Welt der Sterne, die auf ihren ewigen Bahnen den „Einklang“ einer Sphärenmusik und die Harmonie heiliger Naturkräfte erahnen lassen, findet sich häufig in Alexander von Humboldts Schriften. So tritt dem modernen (gespaltenen) Subjekt die kosmische Natur als jenes Reich der Freiheit entgegen, das ihm zumindest zeitweise die Freiheit von der (Menschheits-)Geschichte gewährt. Die stetige Beschleunigung, die sich in Humboldts Text bemerkbar macht, wird nicht in Bewegungslosigkeit, wohl aber in die Synchronie einer sich nicht verändernden sphärischen Bahn der Himmelskörper zurückgeführt. Alles in diesem Kosmos ist Ordnung und Schönheit zugleich.

Wie der Mensch im diesem Kosmos, so scheinen die verschiedenen Textinstanzen (einschließlich der wissenschaftlichen) letztlich in der philosophischen Instanz aufgehoben zu sein, die auf das Ganze gerichtet ist und daher jene alles zusammenführende, synthetisierende Denk-Figur verkörpert, die für Georg Forster wie für Alexander von Humboldt von alles entscheidender Bedeutung war. Humboldt folgt hierin den Spuren Forsters und geht über dessen Entwurf zugleich weit hinaus. Doch sollten wir uns noch einmal das Zusammenspiel der verschiedenen Textinstanzen innerhalb der Ansichten der Natur vergegenwärtigen, bevor wir hieraus weitergehende Schlüsse für die Relevanz der Humboldt'schen Schreibweise im Kontext der Relationen zwischen Reisen und Schreiben ziehen. 
Auch in diesem Bereich sparte Humboldt durchaus nicht an gesellschaftlicher Kritik. In seiner auf März 1849 datierten Vorrede zur zweiten und dritten Ausgabe betonte er die von ihm angestrebte poetologisch relevante „Verbindung eines litterarischen und eines rein scientifischen Zweckes“ und verband diese Bemerkung mit einer unüberhörbaren Kritik am „dogmatischen Halbwissen wie der vornehmen Zweifelsucht“, die in den „sogenannten höheren Kreisen des geselligen Lebens“ verbreitet seien. ${ }^{31}$ Diese Kritik von einem Achtzigjährigen, der bei Hofe als Kammerherr diente und in das gesellschaftliche Leben Berlins noch immer gut integriert war, saß ohne Zweifel.

Humboldt war sich immer seines Lesepublikums sehr bewusst. Er habe seine Schrift nach den „Bedürfnissen der Zeit ganz umzuschmelzen“32 gesucht, eine Metaphorik, die er bereits in seiner Vorrede zur ersten Ausgabe verwendet hatte, als er von den unterschiedlichen Orten des Schreibens und deren Beziehung zum Reisen sprach:

Einzelne Fragmente wurden an Ort und Stelle niedergeschrieben, und nachmals nur in ein Ganzes zusammengeschmolzen. Ueberblick der Natur im Grossen, Beweis von dem Zusammenwirken der Kräfte, Erneuerung des Genusses, den die unmittelbare Ansicht dem fühlenden Menschen gewährt - sind die Zwecke, nach denen ich strebte. Jeder Aufsatz sollte ein in sich geschlossenes Ganzes ausmachen, in allen sollte Eine und dieselbe Tendenz sich gleichmässig zeigen. Die ästhetische Behandlung naturhistorischer Gegenstände hat, trotz der herrlichen Kraft und Biegsamkeit unserer vaterländischen Sprache, grosse Schwierigkeiten der Composition. Der Reichtum der Natur veranlasst Anhäufung einzelner Bilder. Diese Anhäufung aber stört die Ruhe und den Totaleindruck des Naturgemäldes. ${ }^{33}$

Wieder spricht Humboldt vom anzustrebenden 'Naturgemälde' und damit von der Modellhaftigkeit des von ihm Darzustellenden im Lichte einer Verschmelzung von Kunst und Wissenschaft. Er erkannte insbesondere die Gefahr, dass „der Styl leicht in eine dichterische Prosa“ ausarten könnte. ${ }^{34}$ Wie war dieser Gefahr zu begegnen? Klar und offenkundig ist nur, dass sich Humboldt auch beim Schreiben seiner Ansichten der Natur der Amerikanischen Reisetagebücher bediente, die sein eigenes Schreiben im Angesicht der Dinge gleichsam in die literarische Bearbeitung weitergaben. Die Amerikanischen Reisetagebücher sind folglich in ihrer Bedeutung gar nicht zu überschätzen.

31 Ich zitiere hier nach der leicht zugänglichen und die Schreibweise Humboldts nicht unnötig modernisierenden Ausgabe von Humboldt, Alexander von: Ansichten der Natur, mit wissenschaftlichen Erläuterungen. Nördlingen: Greno 1986, S. 9 f.

32 Ebda., S. 10.

33 Humboldt, Alexander von: Ueber Steppen und Wüsten, S. V-VI.

34 Ebda., S. 10. 
Seiner schriftstellerischen Herausforderung und den „Schwierigkeiten der Composition“ suchte er ebenso wie der Gefahr der nur additiven Akkumulation durch die Schaffung einer philosophischen Instanz zu begegnen, die in ihren Einsichten und Ansichten wie in ihrer Metaphorik gleichsam an den Kosmos, an eine Totalität von Himmel und Erde, rückgebunden wird. In ihr ist das 'Literarische' wie das 'Wissenschaftliche' aufgehoben, in ihr kommt es zur Überführung der Linearität sukzessiv angehäufter Bilder in eine quasi-simultane Wahrnehmung eines Naturgemäldes, das Humboldt in den Ansichten der Natur nicht mit den Mitteln der Pasigraphie, sondern mit ästhetischen Verfahren als „Totaleindruck“ zu schaffen strebt.

Mit der philosophischen Instanz allein aber war eine Verbindung des 'Literarischen' mit dem 'Wissenschaftlichen' nicht zu bewerkstelligen. Die Gefahr bestand in der Wahrnehmung als 'Naturphilosophie', mit welcher Humboldt freilich lange schon gebrochen hatte. Welchen Weg also wählte Humboldt, um eine vor Missverständnissen geschützte Vielverbundenheit aller reiseliterarischen Teile seiner Ansichten der Natur sicherzustellen?

Daher sei abschließend ein weiterer, bislang unerwähnt gebliebener Abschnitt aus 'Ueber die Steppen und Wüsten' näher untersucht, um Aufschluss über spezifisch literarische Vermittlungsformen zwischen beiden Bereichen zu erhalten. Vorrangig soll untersucht werden, wie gleichsam unter dem Dach der philosophischen Instanz das von Humboldt stets betonte „Zusammenwirken der Kräfte“ ästhetisch gestaltet werden konnte. Dies sei am Beispiel des eher selten diskutierten Phänomens der Sandhosen erläutert:

\footnotetext{
Wenn unter dem senkrechten Stral der nie bewölkten Sonne die verkohlte Grasdecke in Staub zerfallen ist, klaft der erhärtete Boden auf, als wäre er von mächtigen Erdstößen erschüttert. Berühren ihn dann entgegengesetzte Luftströme, und pflanzt sich durch Gegenstoß die kreisende Bewegung fort; so gewährt die Steppe einen seltsamen Anblick. Als trichterförmige Wolken, deren Spitzen an der Erde hingleiten, steigt der Sand dampfartig durch die luftdünne, vielleicht elektrisch geladene, Mitte des Wirbels empor - gleich den rauschenden Wasserhosen, die der erfahrene Schiffer fürchtet. Ein trübes, strohfarbiges Halblicht wirft die nun scheinbar niedrigere Himmelsdecke auf die verödete Flur. Der Horizont tritt plötzlich näher. Er verengt die Steppe, wie das Gemüth des Wanderers. Die heiße, staubige Erde, die im nebelartig verschleierten Dunstkreise schwebt, vermehrt die erstickende Luftwärme. Statt Kühlung führt der Ostwind neue Gluth herbei, wenn er über den langerhitzten Boden hinweht. ${ }^{35}$
}

Alexander von Humboldt stand vor der Aufgabe, seinem Lesepublikum möglichst wissenschaftlich korrekt das Phänomen einer Sandhose zu erläutern, dabei aber seinen scientifischen Zweck mit literarischen Mitteln zu verbinden

35 Humboldt, Alexander von: Ueber die Steppen und Wüsten, S. $29 \mathrm{f}$. 
und in einen ästhetisch wirkungsvollen reiseliterarischen Text umzusetzen. Wie ging er vor? Die naturwissenschaftlich fundierte Erklärung des Phänomens der Sandhose wird durch die Einführung des Zusammentreffens einer vertikalen mit einer horizontalen Achse eingeleitet, wobei die erstgenannte, der senkrechte Strahl der Sonne, nicht nur zerstörerisch die Vegetation in Staub verwandelt und in den Boden eindringt, sondern Erdrisse erzeugt, wie sie von einer Macht des Erdinnern hervorgerufen sein könnten. Humboldt geht, wie sich zeigt, systematisch und geradezu strukturalistisch vor.

Die Vertikalität des Sonnenstrahls - dessen Intensität Humboldt faszinierte und die er durch Messungen genauer zu fassen suchte - überträgt sich eher metonymisch als kausal auf den trichterförmigen Wirbel, der die Ebene quert und dabei eine nun aufsteigende Bewegung produziert, die der ursprünglich zugeführten Energierichtung entgegengesetzt ist. Es kommt zu einer Zirkulation, zu einer Wechselwirkung. In diese kraftvoll narrativ entfaltete Passage wird durch den Vergleich mit dem Phänomen der Wasserhose ein semantisches Feld eingeblendet, mit dem wir seit Beginn des Textes vertraut sind, wird hier doch wieder die Horizontalität der Steppe mit jener des Meeres in Beziehung gesetzt.

Zugleich kündigt die Figur des erfahrenen Schiffers bereits jene des Wanderers an, insofern beide in ihrer Durchquerung der Anökumene jenen Naturgewalten und Kräften ausgeliefert sind, die sie in ihrer Vertikalität und zerstörerischen Kraft weit überragen. Die ungeheure Dynamik, die im Text von der Sonne ausgelöst wird, führt $\mathrm{zu}$ einer ständig beschleunigten narrativen Bewegung, die nur kurzzeitig von diskursiven Elementen (etwa den Vergleichen mit den Erdstößen oder dem Meer) unterbrochen wird. Die Kraft der vertikalen Erscheinung verändert nicht nur die scheinbar stabile Horizontalität an sich (das Zerfallen in Staub, das Aufklaffen der Erde usw.), sondern grenzt diese Horizontalität auch ein.

Die Plötzlichkeit des Ereignisses weist darauf hin, dass die Darstellung wohl in den Händen der auktorial gestalteten Erzählinstanz liegt, die von ihr ins Spiel gebrachte Wahrnehmung nun aber beim einsamen Wanderer fokalisiert wird. Dies ist ein für die Humboldt'sche Schreibweise charakteristischer Wechsel. Die Kräfte der Natur wirken nicht nur auf die nach außen gerichtete Wahrnehmung des Wanderers, sondern auch auf seine innere Befindlichkeit, sein „Gemüth“ oder, wie es in der französischen Fassung dieses Textes heißt, auf sein Herz. ${ }^{36}$ Die den Abschnitt beendenden Sätze sind ebenfalls präsentisch gehalten, enthalten noch immer Verben der Bewegung, sind aber gleichwohl stärker erläuternder, diskursiver Natur, wobei die Fokalisierung im Subjekt des Wanderers wieder

36 Vgl. Humboldt, Alexandre de: Tableaux de la Nature, S. 42: „Les limites de l'horizon se rapprochent subitement; la steppe se rétrécit, et le coeur du voyageur se resserre.“ 
aufgegeben wird. Sie diente nur dem Ausdruck und der Ausgestaltung einer subjektiv-modernen Modalität.

Ein naturwissenschaftlich erklärbares Phänomen wird hier offenkundig nicht im Diskurs der eigentlich 'zuständigen' Wissenschaften dargelegt. Humboldt wählt bewusst einen anderen Weg. Die wissenschaftlichen Erläuterungen des Phänomens der Sandhose sind unverkennbar in der Modalität des Narrativen und Erlebnishaften, im Gestus einer modernen Subjektivität oder einer Subjektivität der Moderne, gestaltet. Der Dynamik des Naturphänomens entspricht die Dynamik der Erzählung, in der die diskursiven Elemente der Erläuterung, wo irgend möglich, in die Narration - um die Humboldt'sche Metaphorik aufzunehmen - eingeschmolzen sind.

Die Spannungskurve erreicht dabei am Ende des zweiten Drittels dieses Abschnitts ihren Höhepunkt, wobei sich nun die Spannung nicht mehr vorwiegend zwischen der wissenschaftlichen Instanz und der auktorial gestalteten Erzählinstanz des erinnernden Ich, sondern zwischen letzterer und der Perspektive des Wanderers (im Reisebericht also jener des erzählten Ich) aufbaut. Die wissenschaftliche Instanz wird zugleich durch die „wissenschaftlichen Erläuterungen“, die schon im Titel des Buches angekündigt sind, paratextuell gestärkt. Sie wird dabei aber nicht gänzlich auf diese Erläuterungen ausgelagert.

Hierbei handelt es sich freilich nicht nur um zusätzliche Erläuterungen oder - wie in späteren Ausgaben hinzugefügte - Messungsergebnisse, sondern teilweise auch um wahre Erzählkerne, die zu Ausgangspunkten zusätzlicher Erzählvorgänge werden könnten. Denn so, wie der Haupttext von wissenschaftlichen Diskursen gequert wird, so werden auch die Erläuterungen wiederum von narrativen Elementen umsponnen. Auch hier ist alles Wechselwirkung. Die Verbindung eines literarischen mit einem rein szientifischen Zweck ist von Humboldt ernst gemeint.

In der Erstausgabe von 'Ueber die Steppen und Wüsten' umfasst der fortlaufende Text die Seiten 1-46, die „Erläuterungen und Zusätze“ dann die Seiten 46-155. Da sich Humboldts proliferierendes Schreiben in den späteren Ausgaben vor allem in dem letztgenannten paratextuellen Teil entlädt - er modifiziert und erweitert vorhandene Anmerkungen und fügt zusätzliche Anmerkungen hinzu -, pendelt sich die Relation zwischen fortlaufendem Text und wissenschaftlichen Erläuterungen, die in der Erstausgabe bei einem Verhältnis 1: 3 lag, bei der dritten Ausgabe bei einem Verhältnis von 1: 8 ein, ja geht noch bei anderen Texten deutlich darüber hinaus. Vergleichbar ist eine derartige Schreibweise in neuerer Zeit wohl am besten mit jener des großen lateinamerikanischen Bewunderers von Alexander von Humboldt, des kubanischen Anthropologen und Essayisten Fernando Ortiz, der in seinem Contrapunteo cubano del tabaco y el azúcar eine ähnlich 
rhizomatische Schreibweise praktizierte. ${ }^{37}$ Teilt man Schriftsteller in zwei Klassen ein - jene, die bei Korrekturen eher kürzen und jene, die stets hinzufügen -, so gehört Alexander von Humboldt mit seiner Lust an einer proliferierenden Art des Schreibens fraglos der zweiten Klasse an. Es ging ihm um eine ständige Weitung und Erweiterung: und letztlich ums Ganze.

Die Verbindung von 'Literarischem' und 'Wissenschaftlichem' wird in dieser Passage als ein Ineinandergreifen der verschiedenen Instanzen erkennbar, das in der Tat zu einem Zusammenwirken aller Kräfte führt, die auf das Lesepublikum gebündelt einwirken sollen. Die Narrativierung des Diskursiven wird durch eine diskursive Aufladung des Narrativen ergänzt. Auch hier verfährt Humboldt nach dem Prinzip der Wechselwirkung. Die Modernität dieser Schreibweise, die nicht nur die unterschiedlichsten Wissenschaftsbereiche, sondern auch die verschiedenen Textinstanzen miteinander vermittelt, führt zu einer Ganzheit von Darstellung und Erkenntnis, die Wissen und sinnliche Erfahrung unmittelbar aufeinander bezieht.

Humboldts Schreibweise zielt nicht vorrangig auf Eindeutigkeit - obwohl sie diesen Anspruch selbstverständlich keineswegs aufgibt -, sondern erweist sich als bewusst mehrfach kodiert. Diese Mehrfachkodierung ist dabei im engeren Sinne eine literarische. Die „ästhetische Behandlung naturhistorischer Gegenstände“ wird, trotz aller „Schwierigkeiten der Composition“, durch eine Schreibweise eingelöst, die sich nicht als eine bloß literarische, sondern als ein Zusammenfügen aller Elemente zu einem Ganzen versteht. Eine solche écriture erzeugt nicht einfach einen literarischen Mehrwert, sie verwandelt sich vielmehr selbst in einen Wert, der sich nicht als ein Hinzugefügtes, sondern als ein ästhetisch Zusammengefügtes in seiner Komplexität beschreiben lässt.

Wir haben es also nicht mit einer „Anhäufung“, nicht mit Wissenschaft plus Literatur (im rhetorischen Sinne von Schmuck und Ornament), nicht also mit einer additiven Struktur oder Sammlung, sondern mit einer Gesamtheit, die auf den „Totaleindruck“ zielt, zu tun. Diese Ästhetik ist Fundament und Medium der Humboldt'schen Wissenschaftskonzeption zugleich. Sie erzeugt eine Literatur und Wissenschaft in ständiger Bewegung. Die Kräfte, die sie einbindet, entstehen durch Wechselwirkung.

Bei Alexander von Humboldt ergibt sich der recht Aufsehen erregende Befund, dass die Theorien in seinem wissenschaftlichen Werk und dessen reiseliterarische

37 Vgl. Ette, Ottmar: Fernando Ortiz. In: Nünning, Ansgar (Hg.): Metzler Lexikon Literatur- und Kulturtheorie. Ansätze - Personen - Grundbegriffe. Stuttgart - Weimar: Metzler 1998, S. 410-411; sowie (ders.): Transatlantische Transplantationen: Von Pfropfung und 'mestizaje' zum transarchipelischen Zusammenleben in den Amerikas. In: Ette, Ottmar / Wirth, Uwe (Hg.): Kulturwissenschaftliche Konzepte der Transplantation. Berlin - Boston: Walter de Gruyter 2019, S. 27-63. 
Umsetzungen den gleichen Strategien gehorchen und nach denselben Grundsätzen modelliert sind. Ein Buch von der Natur, so gab er zu Protokoll, sollte den Eindruck wie die Natur hervorrufen. Die aufgezeigten Vertextungsstrategien Alexander von Humboldts sind ebenso für die Gesamtheit seiner Schriften wie für die Rezeption seines Werkes von grundlegender, bis heute freilich wenig untersuchter Bedeutsamkeit. Sie ließen sich durch die Analyse zusätzlicher Verfahren seiner reiseliterarischen écriture ergänzen. Von besonderer Wichtigkeit scheinen mir hierbei die von Humboldt stets betonten Beziehungen des Wissens zur sinnlichen Erfahrung der Leserschaft zu sein, so dass an dieser Stelle nicht nur auf die intertextuellen, sondern mehr noch auf die intermedialen Beziehungen hinzuweisen ist.

An dieser Stelle sei lediglich erwähnt, dass insbesondere die Bild-TextBeziehungen, ${ }^{38}$ die sich mannigfaltig in seinen Schriften finden, und die KlangText-Beziehungen, ${ }^{39}$ wie sie etwa in dem ebenfalls in die Ansichten der Natur aufgenommenen Aufsatz über 'Das nächtliche Thierleben im Urwalde' in Szene gesetzt werden, höchste Aufmerksamkeit verdienen. Humboldt war an diesen transmedialen Dimensionen seiner Reisewerke äußerst interessiert, ging es ihm doch darum, die Sinne seines Lesepublikums für die Wahrnehmung seiner die Natur simulierenden Schriften sinnlich zu schärfen. Sinn und Sinnlichkeit des Schreibens bildeten für ihn eine Einheit.

Nur noch ein weiteres Beispiel sei an dieser Stelle für diese Transmedialität angeführt. Die Verschiedenheit der phonotextuellen wie der ikonotextuellen Verfahren erweitert beispielsweise in Humboldts Vues des Cordillères et Monuments des Peuples Indigènes de l'Amérique, seinen Pittoresken Ansichten der Cordilleren, das Spektrum der Wahrnehmungs- und Darstellungsformen beträchtlich, insoweit die inter- und vor allem transmedialen Beziehungen doch jeweils von unterschiedlichen Textinstanzen her organisiert werden. Gerade Humboldts auf Forsters Bemühungen zurückgehender, aber ständig kreativ

38 Vgl. hierzu Ette, Ottmar: Bild-Schrift, Schrift-Bild, Hand-Schrift. Zur Kunst der Sichtbarmachung in Alexander von Humboldts 'Amerikanischen Reisetagebüchern'. In: Ette, Ottmar / Müller, Gesine (Hg.): Visualisierung, Visibilisierung und Verschriftlichung. Schrift-Bilder und Bild-Schriften im Frankreich des 19. Jahrhunderts. Berlin: Verlag Walter Frey - edition tranvía 2015, S. 11-64; sowie ders.: Einführung: Die Bilder-Welten Alexander von Humboldts. Als die Bilder laufen lernten. In: Ette, Ottmar / Maier, Julia: Alexander von Humboldt: Bilder-Welten. Die Zeichnungen aus den Amerikanischen Reisetagebüchern. München: Prestel 2018, S. 9-25.

39 Vgl. hierzu Ette, Ottmar: ,Motion, Emotion, Musik: Alexander von Humboldts experimentelles Schreiben.' Eröffnungsvortrag der Jahrestagung der Schweizerischen Gesellschaft für Allgemeine und Vergleichende Literaturwissenschaft (SGAVL) »Musique et émotions dans la littérature / Musik und Emotionen in der Literatur« an der Universität Bern (16.11.2017, Tagungsband im Druck). 
erweiterter Versuch, die Ganzheit des Erfahrbaren und Verstehbaren nicht nur zu erfassen, sondern auch zu vermitteln, hat ihn nach immer wieder neuen ästhetischen Ausdrucksformen suchen lassen. An dieser Stelle stoßen wir auf die experimentelle Dimension des Humboldt'schen Schreibens, eines reiseliterarischen Schreibens, das sich seiner Zuschauer und Hörerschaft jeweils sehr bewusst war. Seine oftmals selbstreflexiven Briefe an Varnhagen von Ense sind, vergleichbar mit jenen Gustave Flauberts an Louise Colet, ein beredtes Zeichen dieser ruhelosen Suche eines Schreibens in der Moderne.

Gleichwohl gibt es - ungeachtet aller Veränderungen - eine Kontinuität und Kohärenz in der Entwicklung und Ausprägung der Humboldt'schen Schreibweise, die angesichts der sich über sieben Jahrzehnte erstreckenden Veröffentlichungstätigkeit des großen Gelehrten aus heutiger Sicht mehr als nur beeindruckend ist. Denn Humboldt blieb sich über lange Jahrzehnte in vielen Dingen wie auch in seiner konsequenten Weiterentwicklung von Ideen, die er über lange Jahrzehnte verfolgte, treu. Dies ist nicht zuletzt durch seine in ihren Grundlagen unerschütterliche, ethisch fundierte Konzeption von Wissenschaft und allgemeiner noch von Wissen in der Gesellschaft überhaupt bedingt - gerade auch, was die gesellschaftliche Distribution von Wissen angeht.

Wir kommen damit zum Komplex dessen, was wir heute als die Humboldt'sche Wissenschaft bezeichnen. Susan Faye Cannon hat als erste in ihrer wissenschaftshistorischen Untersuchung angesichts der terminologisch nicht auf den Begriff zu bringenden Fülle der von Humboldt behandelten Gegenstände und angewandten Verfahren den im deutschsprachigen Raum zunächst kaum wahrgenommenen Begriff der Humboldtian Science geprägt und hinzugefügt:

Wenn Humboldt ein Revolutionär war (was er nach meiner Ansicht vielleicht tatsächlich war), lag der Grund hierfür nicht darin, dass er all die unterschiedlichen Teile der Humboldt'schen Wissenschaft erfunden hätte. Er war es, weil er den gesamten Komplex für etwa vierzig Jahre zur Hauptbeschäftigung professioneller Wissenschaft erhob. ${ }^{40}$

Mit diesen Worten und mit ihrer Arbeit legte die Wissenschaftshistorikerin eine neue Grundlage für die Sichtweise und das Verständnis Alexander von Humboldts heute. Auch wenn man Alexander von Humboldt weder in politischen noch in wissenschaftlichen Dingen für einen Revolutionär halten muss, ${ }^{41}$ kann man doch der Überzeugung beipflichten, dass der Verfasser der Reise in die Äquinoktial-Gegenden des Neuen Kontinents eine Wissenschaftskonzeption

40 Cannon, Susan Faye: Science in Culture: The Early Victorian Period. New York: Dawson and Science History Publications 1978, S. 77.

41 Vgl. hierzu die Überlegungen in Ette, Ottmar: Mobile Preußen. Ansichten jenseits des Nationalen. Stuttgart: Metzler 2019. 
sui generis begründete, deren Charakteristikum weniger in der Innovation (oder 'Erfindung') als in der Kombination und Verkettung gesehen werden darf. Dabei wissen wir vier Jahrzehnte nach Susan Faye Cannon, dass die Humboldt'sche Wissenschaft in ihrer transdisziplinären, transarealen und lebenswissenschaftlichen Dimensionen noch wesentlich konziser und konsistenter war, als man sich dies ursprünglich vorstellte. Die Existenz einer Humboldt'schen Wissenschaft hatte selbstverständlich auch direkte Konsequenzen für das reiseliterarische Verhältnis von Reisen und Schreiben bei Humboldt.

Schon früh ist die Kombinatorik und das, was Humboldt später als die 'Wechselwirkung' bezeichnete, zu einem Merkmal des 'kleinen Apothekers' von Schloss Tegel geworden, das sein Bruder Wilhelm in einem Brief vom 18. März 1793 an Carl Gustav von Brinkmann wohl erkannte:

Ich halte ihn unbedingt und ohne alle Ausnahme für den größten Kopf, der mir je aufgestoßen ist. Er ist gemacht, Ideen zu verbinden, Ketten von Dingen zu erblicken, die Menschenalter hindurch, ohne ihn, unentdeckt geblieben wären. Ungeheure Tiefe des Denkens, unerreichbarer Scharfblick, und die seltenste Schnelligkeit der Kombination, welches alles sich in ihm mit eisernem Fleiß, ausgebreiteter Gelehrsamkeit, und von unbegränztem Forschungsgeist verbindet, müssen Dinge hervorbringen, die jeder andere Sterbliche sonst unversucht lassen müßte. ${ }^{42}$

Wilhelm von Humboldt wurde damit zum ersten Epistemologen seines Bruders Alexander und erkannte in der Kombination den Denk- und Wissenschaftsstil des Jüngeren, der auch in seinem literarischen Schreibstil zum Ausdruck kommen sollte. In der Kombinatorik, der Verknüpfung von Ideen, hat Wilhelm bei seinem Bruder Alexander folglich einen Denkstil ausgemacht, der später das gesamte mobile Gebäude der Humboldt'schen Wissenschaft durchdringen sollte. Man könnte mit Recht die Innovation des von Alexander geschaffenen Wissenschaftsparadigmas vorrangig in der spezifischen Kombinatorik erblicken, die zwischen den unterschiedlichsten Gegenstandsbereichen, Wissensgebieten und Methodologien Verbindungen herstellt und so das (auf einer Fülle von Einzeluntersuchungen basierende) Zusammendenken als Herzstück Humboldt'scher Wissenschaftskonzeption ausweist. Man hat Humboldt später oft vorgeworfen, er habe sich nicht zwischen Deutsch und Französisch, nicht zwischen Preußen und Frankreich, nicht zwischen Europa und Amerika, nicht zwischen Natur- und Kulturwissenschaften entscheiden können. Doch dieser Vorwurf geht ins Leere. Denn Humboldt war an den Wechselwirkungen interessiert: Er zielte auf das Ganze.

42 Zitiert nach Beck, Hanno (Hg.): Gespräche Alexander von Humboldts. Berlin: AkademieVerlag 1959, S. 6. 
Die Humboldt'sche Wissenschaft ist ohne jeden Zweifel eine transdisziplinäre und zugleich transareal ausgerichtete Wissenschaft und wird als solche auch verstanden. Sie ist jedoch interkulturell und nicht transkulturell, weil sie bewusst von der europäischen Perspektive ausgeht, eine Tatsache, der sich auch die so häufig in Humboldts Texten beobachtbare Figur des europäischen Reisenden verdankt. Allerdings gibt es bereits bei Humboldt bei genauerer Betrachtung auch eine Reihe transkultureller Elemente, die $\mathrm{zu}$ untersuchen sich mehr als lohnt. Sind wir heute möglicherweise an einem Punkte angelangt, von dem aus die ersten eher zaghaften Schritte in Richtung auf diese transkulturelle Wissenschaft unternommen werden, so ist die Humboldt'sche Perspektivierung stets eine europäische, die den Dialog mit anderen Kulturen sucht und an einem interkulturellen Austausch nicht weniger interessiert ist als an Weltwirtschaft, Welthandel und Weltliteratur in einem geradezu 'klassischen', nicht zuletzt auch Ideen der Weimarer Klassik Goethes aufnehmendem Sinne.

Humboldts Wissenschaftskonzeption ist transdisziplinär ausgerichtet, weil sie nicht - wie im Falle der Interdisziplinarität - vom Standpunkt einer ganz bestimmten Disziplin aus den Dialog mit anderen wissenschaftlichen Fachgebieten und die wechselseitige Erhellung unterschiedlich 'disziplinierter' Wissensgebiete sucht. Sein Leitmodell war ein vernetztes und mehr noch immer neu vernetzendes Denken: keines, das in Oppositionen, in unüberbrückbaren Gegensätzen dachte. So siedelte er sich auch jenseits der Berliner Debatte um die Neue Welt an, indem er - was der gemeinsame Nenner von de Pauw und Pernety war - anders als die Denker des 18. Jahrhunderts in Amerika nicht das Andere von Europa erkannte, sondern vielmehr die Zusammenhänge, das Zusammenführende, betonte und die auch auf diesem Gebiet vernetzenden Beziehungen zwischen beiden Weltteilen hervorhob.

Alexander von Humboldt war bestrebt, in möglichst vielen Disziplinen zuhause zu sein, oder besser: sich zwischen den verschiedenen Disziplinen $\mathrm{zu}$ bewegen und seine vie nomade, seinen nomadischen Lebensstil auch auf den Bereich der Wissenschaften auszudehnen. Es ging ihm daher auch niemals um eine Spezialisierung, die nur einen bruchstückhaften Dialog mit anderen Spezialisten zu führen imstande wäre, sondern um ein nomadisches Wissen, das sich dank weltweit ausgedehnter Korrespondentennetze und einer unermüdlichen Arbeitsleistung stets die Möglichkeit offenhielt, von verschiedenen disziplinären Standpunkten aus zugleich zu argumentieren. Die uns heute bedrückenden Grenzen interdisziplinärer (oder wechselseitig 'disziplinierter') Forschung kennt sein Denken nicht. Und auch dies gehört auch heute noch zum Unabgegoltenen der Humboldt'schen Wissenschaftskonzeption.

Die Humboldt-Industry hat in den zweihundertfünfzig Jahren seit seiner Geburt nur mühsam den transdisziplinären und transarelaen Reichtum eines 
Forschungsunternehmens erkannt, das sich nicht einem einzigen Wissenschaftsverständnis zuordnen lässt. Auf diesem Gebiet ist das hochgelobte Buch von Andrea Wulf sicherlich ein Rückschritt, macht es doch Humboldt wieder zu jenem Naturforscher und Naturwissenschaftler, der er sicherlich auch war, lässt aber die Hälfte der Humboldt'schen Wissenschaft ganz einfach weg. Humboldts Reisen waren Reisen in Natur und Kultur der von ihm bereisten Länder und Regionen, sie waren transdisziplinär und interkulturell sowie vor allem transareal ausgerichtet und zielten auf die Erhellung all jener Wechselwirkungen gerade auch zwischen Natur und Kultur, die für seine Wissenschaftskonzeption tragend waren.

Es dreht sich bei Humboldt um Überzeugungen, Bewegungen und Werte, die nicht nur von biographischer oder wissenschaftshistorischer Bedeutung sind, sondern auch von großer Aktualität und Wichtigkeit für die heutigen Debatten um die Beziehungen sowohl zwischen den verschiedenen Wissenschaften untereinander als auch zwischen diesen und der Gesellschaft innerhalb eines weltweiten Kommunikationszusammenhanges.

Vor allem aber: Die Humboldtian Science ist in einem Humboldtian Writing fundiert, das es dem Verfasser des Examen critique erst erlaubte, mit Hilfe ganz bestimmter Schreibverfahren und Vertextungsstrategien jenen Gesamteindruck zu erzeugen, der auf der Konzeption eines Ganzen, des Ganzen, beruhte. Denn Alexander von Humboldt verstand es, seine Unruhe, seine ständige Beschäftigung mit mehreren Gegenständen zugleich nicht zu einem wissenschaftlichen Dilettantismus verkommen $\mathrm{zu}$ lassen, sondern in einen Prozess ständig zunehmender Komplexität des Wissens und des Schreibens zu verwandeln. Dieser Prozess war bei ihm ein Leben lang unabgeschlossen, in ständiger Bewegung.

Eine Humboldt'sche Wissenschaft wäre ohne ein Humboldt'sches Schreiben unvorstellbar, wäre es dem preußischen Gelehrten ansonsten doch niemals möglich gewesen, das Zusammengedachte auch als solches - und nicht etwa als Zusammengeschriebenes - zu präsentieren. In diesem Humboldt'schen Schreiben, so scheint mir, liegt nicht nur der Schlüssel zu seinem gesamten Werk, sondern auch für ein adäquates Verständnis der Komplexität seiner Vorstellungswelt. Von einzelnen Disziplinen her - und seien dies auch Querschnittsdisziplinen wie die Geographie - ist dieses Verständnis heute nicht mehr zu gewinnen. Die Erfindung der Natur - um bei der Terminologie von Andrea Wulf zu bleiben - macht nur Sinn im Rahmen ihrer Vernetzung mit einer Erfindung der Kultur. Bei Humboldt sind Natur und Kultur unauflöslich miteinander verwoben.

Aber kommen wir nochmals zurück auf das Verhältnis von Reisen und Schreiben in Humboldts Reiseschreiben. Bislang wurden die Schriften Alexander von Humboldts überwiegend so gelesen, als ob das Schreiben, die Form ihrer Präsentation, gleichsam transparent wäre und uns eine unmittelbare 
Kommunikation mit den von ihm jeweils dargestellten Inhalten erlauben könnte. Humboldts eigene Schreibstrategien trugen viel zu dieser Fiktion von Direktheit und Unmittelbarkeit bei, zielten sie doch auf eine möglichst 'natürliche' Wahrnehmung, die Natur gleichsam simulierte. Das Schreiben sollte wie die Natur - oder vielmehr: wie Humboldts kulturell bedingte Sichtweise von Natur - sein. So schrieb er an Varnhagen von Ense:

Dem Oratorischen muss das einfach und wissenschaftlich Beschreibende immerfort gemischt sein. So ist die Natur selbst. Die funkelnden Sterne erfreuen und begeistern, und doch kreist am Himmelsgewölbe alles in mathematischen Figuren. Die Hauptsache ist, dass der Ausdruck immer edel bleibe, dann fehlt der Eindruck von der Größe der Natur nicht. $^{43}$

Alexander von Humboldt ging äußerst behutsam mit jenen literarischen Formen um, die er für sein Reiseschreiben auswählte. Er war sich dabei stets seines Publikums bewusst und versuchte, ebenso in deutscher wie in französischer Sprache gleichsam 'Naturformen' für seine stilistische Ausgestaltung zu finden. Der Verfasser der Ansichten der Natur unternahm dabei zeit seines Lebens immer wieder andere Versuche, einen Effekt der quasi-natürlichen Unmittelbarkeit zu erzielen, ohne Gefahr zu laufen, seine literarische Darstellung als „dichterische Prosa“ gebrandmarkt zu sehen. „Ein Buch von der Natur“, so schrieb er schon am 24. Oktober 1834 an Varnhagen von Ense, „muss den Eindruck wie die Natur selbst hervorbringen“44 - ein Leitthema, das wir bereits angeführt hatten. Der von Humboldt angestrebte literarische Kode sollte gleichsam 'natürlich' überdeterminiert sein, um über seine eigene Kodierung, seine eigene Literarizität hinwegzutäuschen und wie jene Glasscheibe zu wirken, durch die wir - ohne sie zu reflektieren - die Außenwelt betrachten. Und mit Blick auf die Reaktionen seiner Zeitgenossen darf man wohl sagen, dass diese Versuche gelangen.

Freilich handelt es sich hierbei um ein überaus effektives und wohlkalkuliertes Verfahren, das 'Literarische' der Schrift in der Repräsentation der Natur zu tilgen. Humboldt versuchte, den Artefaktcharakter seiner Veröffentlichungen mit Hilfe literarischer Techniken zu verbergen, die - ähnlich wie die Kodes der europäischen Realisten des 19. Jahrhunderts - auf einen 'Realitätseffekt', einen effet de réel, abstellten, wie ihn Roland Barthes definierte. Dem wissenschaftlichen Charakter seiner Schriften tat dies keinen Abbruch. Gegenstände, Inhalte und Ergebnisse seines Denkens wie der von ihm betriebenen Wissenschaften nehmen

43 Briefe Alexander von Humboldts an Varnhagen von Ense, S. 92 (Brief vom 28. April 1841). 44 Ebda., S. 23 (die Datierung auf den 27.10. ist wohl fehlerhaft); vgl. auch Blumenberg, Hans: Die Lesbarkeit der Welt. Frankfurt am Main: Suhrkamp 1986, S. 283 f. 
wir aber nur durch seine Schriften und damit als Ergebnisse überaus komplexer Schreib-, Lese und Darstellungsprozesse wahr. Schon in seinen Reisetagebüchern hat Humboldt dieses Verhältnis zwischen Reisen und Schreiben literarisch höchst kunstvoll ausgeführt.

$\mathrm{Zu}$ lange, so scheint mir, hat die Humboldt-Forschung die Gläser nicht gesehen, durch die sie auf Humboldts Werk blickte. Es gibt noch viel zu erforschen mit Blick auf Humboldt als deutschem und französischem Schriftsteller. ${ }^{45}$ Wenn wir nach den Debatten des 20. und beginnenden 21. Jahrhunderts aus der Sicht verschiedenster Einzeldisziplinen wissen, dass Alexander von Humboldt zweifellos Naturforscher (im Sinne des französischen naturaliste), Historiker, Kulturforscher, Philosoph und Geograph war, so sollten wir heute umso bewusster zur Kenntnis nehmen, dass er sich nicht von Einzeldisziplinen her begreifen lässt und eben mehr war als Kulturforscher, Geograph, Philosoph, Naturforscher oder Historiker. Wenn wir Alexander von Humboldt als literarischen Autor, als Schriftsteller begreifen, so kann es heute jedoch nicht mehr darum gehen, nunmehr ausgehend von der 'Schriftstellerei' - die freilich keine Disziplin, sondern eher ein bestimmter Typus von Aktivität ist - den Verfasser des Kosmos zu vereinnahmen. Doch eine tiefgehendere Beleuchtung des Humboldtian Writing ist dringend erforderlich.

Hierbei geht es sehr wohl um die Frage, mit Hilfe welcher Verfahren und Techniken es Humboldt gelang, die komplexe Kombinatorik eines transdisziplinären Forschens und interkulturellen Denkens zu re-präsentieren und zugleich ein grenzüberschreitendes Denken und Schreiben in beständiger Bewegung zu halten. Es geht um eine Neubewertung des bei ihm beobachtbaren Verhältnisses von Reisen und Schreiben. Dabei spielen seine Reisetagebücher und insbesondere seine Amerikanischen Reisetagebücher eine ganz entscheidende Rolle. Es war just diese Leistung des Humboldt'schen Schreibens 'im Angesicht der Dinge', welche die unabdingbare Voraussetzung der Humboldt'schen Wissenschaft bildete.

Dabei sollten wir freilich nicht vergessen, dass für Alexander von Humboldt Wissenschaft, Ethik und Ästhetik ein unauflösbares Ganzes bildeten und dass ihn, wie er in Mes confessions bekannte, eine „inquiétude morale“ umtrieb, für die auch die Grenzen des Transdisziplinären bei weitem zu eng gesteckt gewesen wären. Die für Humboldts Denken grundlegende Verbindung von Ethik und Ästhetik lässt sich nicht nur auf die Figur des 'nomadisierenden' Reisenden beziehen, dem als Vermittlungsinstanz kultureller Alteritätserfahrung eine

45 Vgl. hierzu Lenz, Markus Alexander: Französische Literaten. In Ette, Ottmar (Hg.): Alexander von Humboldt-Handbuch. Leben - Werk - Wirkung. Mit 52 Abbildungen. Stuttgart: J.B. Metzler Verlag 2018, S. 229-235. 
gattungskonform zentrale Funktion zukommt, sondern erlaubt auch Ausblicke auf eine sich abzeichnende Figur des Intellektuellen, der nicht an die Grenzen wissenschaftlicher Diskurse gebunden ist. Humboldt war in diesem ethisch fundierten Sinne ganz ohne Zweifel ein Intellektueller avant la lettre, der sich seine Unabhängigkeit, ungeachtet seiner Dienste als Kammerherr bei Hofe, zu erhalten wusste und auch in seiner privaten Korrespondenz - wie die Briefe mit Varnhagen von Ense zeigen - behielt.

Denn das Schreiben in der Moderne schließt für Alexander von Humboldt ganz wie bereits für Georg Forster - stets eine Verantwortung ein, die wir heute als eine Funktion des Intellektuellen begreifen. Auch diese öffentliche Funktion in und für die Gesellschaft zählt zum Kernbestand dieser europäischen Moderne, für die Humboldts Schreiben stellvertretend stehen kann. So weitgefächert auch immer seine Wissenschaftskonzeption sein mochte, er sah sie stets wie auch sich selbst in der gesellschaftlichen Verantwortung. Und mehr noch: Sie war für ihn ein Motor der gesamtgesellschaftlichen Entwicklung im Sinne nicht nur einer Popularisierung wissenschaftlicher Themen, sondern mehr noch einer Demokratisierung des Zugangs zu Wissen und dessen Zirkulation.

Auch hierin liegt die Modernität eines Schreibens, das die perspektivische Vielgestaltigkeit der europäischen Moderne, an deren Herausbildung Humboldt wesentlich mitgearbeitet hat (und an deren Ende wir uns möglicherweise befinden), wie kaum ein anderes zum Ausdruck brachte. Mit Alexander von Humboldt erfährt die europäische Reiseliteratur einen ungeheuren Schub und zugleich eine nunmehr moderne, die Subjektivität der Reisenden vielfach beleuchtende Modellierung.

Das Humboldtian Writing ist ein Reiseschreiben in der Moderne, das über diese weit hinausreichend nichts von seiner ästhetischen Kraft und seiner moralischen Unruhe für uns heute verloren hat. Es zeigt und setzt Literatur in mehrfachem Sinne in Bewegung und entfaltet eine Dynamik, die in der Heterogenität ihrer diskursiven Brennstoffe aus heutiger Perspektive wieder genauer wahrgenommen werden kann. Mit Humboldt erreicht das europäische Reiseschreiben einen Höhepunkt, der für viele nachfolgende Reisen, für viele Autorinnen und Autoren als reiseliterarischer Bezugspunkt wirkte. 